\title{
ON INTERSECTIONS OF CERTAIN PARTITIONS OF A GROUP COMPACTIFICATION
}

\author{
XUHUA HE AND JIANG-HUA LU
}

\begin{abstract}
Let $G$ be a connected semi-simple algebraic group of adjoint type over an algebraically closed field, and let $\bar{G}$ be the wonderful compactification of $G$. For a fixed pair $\left(B, B^{-}\right)$of opposite Borel subgroups of $G$, we look at intersections of Lusztig's $G$-stable pieces and the $B^{-} \times B$-orbits in $\bar{G}$, as well as intersections of $B \times B$-orbits and $B^{-} \times B^{-}$-orbits in $\bar{G}$. We give explicit conditions for such intersections to be non-empty, and in each case, we show that every non-empty intersection is smooth and irreducible, that the closure of the intersection is equal to the intersection of the closures, and that the non-empty intersections form a strongly admissible partition of $\bar{G}$.
\end{abstract}

\section{INTRODUCTION}

1.1. Let $Z$ be an irreducible algebraic variety over an algebraically closed field k. By a partition of $Z$ we mean a finite disjoint union $Z=\bigsqcup_{i \in \mathcal{I}} X_{i}$ such that each $X_{i}$ is a smooth irreducible locally closed subset of $Z$ and that the closure of each $X_{i}$ in $Z$ is the union of some $X_{i^{\prime}}$ 's for $i^{\prime} \in \mathcal{I}$.

1.2. Let $G$ be a connected semi-simple algebraic group of adjoint type over an algebraically closed field k. Regard $G$ as a $G \times G$ homogeneous space by the action

$$
\left(g_{1}, g_{2}\right) \cdot g=g_{1} g g_{2}^{-1}, \quad g_{1}, g_{2}, g \in G .
$$

The De Concini-Procesi wonderful compactification $\bar{G}$ of $G$ is a smooth $(G \times G)$-equivariant compactification of $G$ [2, 3].

Let $B$ and $B^{-}$be a pair of opposite Borel subgroups of $G$. The partition of $\bar{G}$ into the $B \times B$-orbits was studied in [1] and [27]. In his study of parabolic character sheaves on $\bar{G}$ in [22, 23], G. Lusztig introduced a decomposition of $\bar{G}$ into finitely many $G$-stable pieces, where $G$ is identified with the diagonal $G_{\text {diag }}$ of $G \times G$. It was later proved in [12] that Lusztig's $G$-stable pieces form a partition of $\bar{G}$.

This paper concerns with

1) intersections of $B \times B$-orbits and $B^{-} \times B^{-}$-orbits in $\bar{G}$,

2 ) intersections of the $G$-stable pieces with $B^{-} \times B$-orbits in $\bar{G}$.

Our motivation partially comes from Poisson geometry. Let $H=$ $B \cap B^{-}$. When $\mathbf{k}=\mathbb{C}$, there is [6, 21] a natural $H \times H$-invariant Poisson 
structure $\Pi_{1}$ on $\bar{G}$ whose $H \times H$-orbits of symplectic leaves are the nonempty intersections of $B \times B$ and $B^{-} \times B^{-}$-orbits. Similarly, there is natural $H_{\text {diag }}$-invariant Poisson structure $\Pi_{2}$ on $\bar{G}$ whose $H_{\text {diag-orbits }}$ of symplectic leaves are the non-empty intersections of $G_{\text {diag }}$-orbits and $B^{-} \times B$-orbits. The restrictions of $\Pi_{1}$ and $\Pi_{2}$ to $G \subset \bar{G}$ are closely related to the quantized universal enveloping algebra of the Lie algebra of $G$ and its dual (as a Hopf algebra). See [7, 19].

The closures of such intersections also appear in the study of algebrogeometric properties of $\bar{G}$. In the joint work [16] of He and Thomsen, it was proved that in positive characteristics, there exists a Frobenius splitting on $\bar{G}$ which compatibly splits all the nonempty intersections of the closures of $B \times B$-orbits and $B^{-} \times B^{-}$-orbits in $\bar{G}$. In particular, all such closures are weakly normal and reduced. Moreover, the closure of a $B \times B$-orbit is globally F-regular in positive characteristic and is normal and Cohen-Macaulay for arbitrary characteristic.

Later, in the joint work [17] of He and Thomsen, it was proved that in positive characteristics, there exists a Frobenius splitting on $\bar{G}$ which compatibly splits all the nonempty intersections of the closures of $G$ stable pieces and $B^{-} \times B$-orbits in $\bar{G}$. In particular, all such closures are weakly normal and reduced. However, the closure of a $G$-stable piece is not normal in general [17, No. 11.2].

1.3. To state our results more precisely, we introduce some notation. Let $N_{G}(H)$ be the normalizer of $H$ in $G$, and let $W=N_{G}(H) / H$ be the Weyl group. Let $\Gamma$ be the set of simple roots determined by the pair $(H, B)$. For $J \subset \Gamma$, let $W_{J}$ be the subgroup of $W$ generated by $J$, and let $W^{J} \subset W$ the set of minimal length representatives of $W / W_{J}$ in $W$. If $J^{\prime}$ is a another subset of $\Gamma$, and $x \in W$, let $\min \left(W_{J^{\prime}} x W_{J}\right)$ and $\max \left(W_{J^{\prime}} x W_{J}\right)$ be respectively the unique minimal and maximal length elements in the double coset $W_{J^{\prime}} x W_{J}$.

For $x, y \in W$, let $x * y \in W$ be such that $B(x * y) B$ is the unique dense $(B, B)$-double coset in $B x B y B$. The operation $*$ makes $W$ into a monoid which will be denoted by $(W, *)$. See [25].

Let $\delta \in \operatorname{Aut}(G)$ be such that $\delta(H)=H$ and $\delta(B)=B$. Let $G_{\delta}=$ $\{(g, \delta(g)): g \in G\} \subset G \times G$ be the graph of $\delta$. We will in fact work with $G_{\delta}$-stable pieces in $\bar{G}$ (see definition below).

Recall that the $G \times G$-orbits in $\bar{G}$ are in one to one correspondence with subsets of $\Gamma$. For $J \subset \Gamma$, let $Z_{J}$ be the corresponding $G \times G$ orbit in $\bar{G}$. One has $\overline{Z_{J}}=\bigsqcup_{K \subset J} Z_{K}$, and $\overline{Z_{J}}$ is smooth. See [2, 3]. Let $h_{J}$ be a distinguished point in $Z_{J}$ (see §3.1). For $w \in W^{J}$ and 
$(x, y) \in W^{J} \times W$, let

$$
\begin{aligned}
& Z_{J, \delta, w}=G_{\delta}(B \times B)(w, 1) \cdot h_{J}, \\
& {[J, x, y]=(B \times B)(x, y) \cdot h_{J},} \\
& {[J, x, y]^{-,+}=\left(B^{-} \times B\right)(x, y) \cdot h_{J},} \\
& {[J, x, y]^{-,-}=\left(B^{-} \times B^{-}\right)(x, y) \cdot h_{J} .}
\end{aligned}
$$

The $Z_{J, \delta, w}$ 's are called the $G_{\delta^{-}}$stable pieces in $\bar{G}$. By [12, 27], one has the following partitions of $\bar{G}$ :

$$
\begin{aligned}
\bar{G} & =\bigsqcup_{J \subset \Gamma,(x, y) \in W^{J} \times W}[J, x, y]=\bigsqcup_{J \subset \Gamma,(x, y) \in W^{J} \times W}[J, x, y]^{-,-} \\
& =\bigsqcup_{J \subset \Gamma,(x, y) \in W^{J} \times W}[J, x, y]^{-,+}=\bigsqcup_{J \subset \Gamma, w \in W^{J}} Z_{J, \delta, w} .
\end{aligned}
$$

For a subset $X$ of $\bar{G}$, let $\bar{X}$ be the Zariski closure of $X$ in $\bar{G}$.

We prove (see Proposition 3.1, Theorem 3.2, and Theorem 3.3) that for any $J \subset \Gamma, w \in W^{J}$, and $(x, y),(u, v) \in W^{J} \times W$,

1) $[J, x, y] \cap[J, u, v]^{-,-} \neq \emptyset$ if and only if $x \leqslant u$ and $v \leqslant \max \left(y W_{J}\right)$, and in this case, $[J, x, y] \cap[J, u, v]^{-,-}$is smooth and irreducible, and

$$
\overline{[J, x, y] \cap[J, u, v]^{-,-}}=\overline{[J, x, y]} \cap \overline{[J, u, v]^{-,-}} .
$$

2) $Z_{J, \delta, w} \cap[J, x, y]^{-,+} \neq \emptyset$ if and only if $\min \left(W_{J} \delta(w)\right) \leqslant y^{-1} * \delta(x)$, and in this case, $Z_{J, \delta, w} \cap[J, x, y]^{-,+}$is smooth and irreducible, and

$$
\overline{Z_{J, \delta, w} \cap[J, x, y]^{-,+}}=\overline{Z_{J, \delta, w}} \cap \overline{[J, x, y]^{-,+}} .
$$

Let

$$
\begin{array}{cl}
\mathcal{J}=\{(J, x, y, u, v): & J \subset \Gamma,(x, y),(u, v) \in W^{J} \times W, \\
& \left.x \leqslant u, v \leqslant \max \left(y W_{J}\right)\right\}, \\
\mathcal{K}=\{(J, w, x, y): \quad & J \subset \Gamma,(w, x, y) \in W^{J} \times W^{J} \times W, \\
& \left.\min \left(W_{J} \delta(w)\right) \leqslant y^{-1} * \delta(x)\right\} .
\end{array}
$$

One then has two more partitions of $\bar{G}$ :

$$
\begin{aligned}
\bar{G} & =\bigsqcup_{(J, x, y, u, v) \in \mathcal{J}}[J, x, y] \cap[J, u, v]^{-,-} \\
& =\bigsqcup_{(J, w, x, y) \in \mathcal{K}} Z_{J, \delta, w} \cap[J, x, y]^{-,+}
\end{aligned}
$$

We introduce the notion of admissible partitions and strongly admissible partitions of $\bar{G}$ (see Definition 3.1) and show that the six partitions in (11) and (2) are all strongly admissible (Proposition 3.2, Theorem 3.2, and Theorem (3.3). Moreover, the first two partitions in (11), as well as the last two in (1), are shown to be compatible. As consequences, we prove 
1) if $J \subset \Gamma$ and if $X$ is a subvariety of $Z_{J}$ appearing in any of the six partitions in (11) and (2), then for any $K \subset J, \bar{X} \cap Z_{K} \neq \emptyset$, and $\bar{X}$ and $\overline{Z_{K}}$ intersect properly in $\overline{Z_{J}}$. Moreover, we describe the irreducible components of $\bar{X} \cap \overline{Z_{K}}$ in each case (Corollaries 3.3 and 3.4). This result for $\bar{G}=\bigsqcup_{J \subset \Gamma,(x, y) \in W^{J} \times W}[J, x, y]^{-,+}$was also obtained by M. Brion [1];

2) if $X=[J, x, y]$ and $Y=[K, u, v]^{-,-}$with $J, K \subset \Gamma,(x, y) \in$ $W^{J} \times W$, and $(u, v) \in W^{K} \times W$, or if $X=Z_{J, \delta, w}$ and $Y=[K, x, y]^{-,+}$ with $J, K \subset \Gamma, w \in W^{J}$ and $(x, y) \in W^{K} \times W$, and if $\bar{X} \cap \bar{Y} \neq \emptyset$, we show that $\bar{X}$ and $\bar{Y}$ intersect properly in $\overline{Z_{J \cup K}}$ (Corollary 3.5).

In positive characteristic, let $G_{F}=\{(g, F(g)): g \in G\}$ be the graph of Frobenius morphism $F: G \rightarrow G$. In \$4, we study the intersection of the $G$-stable pieces with $G_{F}$-orbits. Such intersections include as a special case the Deligne-Lusztig varieties.

Our discussions in this paper, and especially that in $\$ 2.9$ and $\$ 3.5$, also apply to intersections of $R$-stable pieces and $B \times B$-orbits, where $R$ is a certain class of connected subgroups of $G \times G$ as in [20], as long as $R \cap(B \times B)$ is connected and that $\operatorname{Lie}(R)+\operatorname{Lie}(B \times B)=\operatorname{Lie}(G \times G)$.

1.4. We set up more notation for the rest of the paper.

For $\alpha \in \Gamma$, let $U^{\alpha}$ be the one dimensional unipotent subgroup of $G$ defined by $\alpha$. For a subset $J$ of $\Gamma$, let $P_{J}$ and $P_{J}^{-}$be respectively the standard parabolic subgroups of $G$ determined by $J$ that contain $B$ and $B^{-}$, and let $U_{J}$ and $U_{J}^{-}$be respectively the uniradicals of $P_{J}$ and $P_{J}^{-}$. Let $M_{J}=P_{J} \cap P_{J}^{-}$be the common Levi factor of $P_{J}$ and $P_{J}^{-}$, and let $\operatorname{Cen}\left(M_{J}\right)$ be the center of $M_{J}$.

The longest element in $W$ is denoted by $w_{0}$. If $J \subset \Gamma$, denote by $w_{0}^{J}$ the longest element in $W_{J}$, and let ${ }^{J} W=\left\{w^{-1}: w \in W^{J}\right\}$. If $J^{\prime}$ is a another subset of $\Gamma$, let ${ }^{J^{\prime}} W^{J}={ }^{J^{\prime}} W \cap W^{J}$.

Throughout the paper, $\bigsqcup$ always means disjoint union.

\section{InterseCtions in $Z_{\mathcal{C}}=(G \times G) / R_{\mathcal{C}}$}

2.1. Following [20], an admissible quadruple for $G$ is a quadruple $\mathcal{C}=$ $\left(J, J^{\prime}, c, L\right)$, where $J$ and $J^{\prime}$ are subsets of $\Gamma, c: J \rightarrow J^{\prime}$ is a bijective map preserving the inner products between the simple roots, and $L$ is a connected closed subgroup of $M_{J} \times M_{J^{\prime}}$ of the form

$$
L=\left\{\left(m, m^{\prime}\right) \in M_{J} \times M_{J^{\prime}}: \theta_{c}(m C)=m^{\prime} C^{\prime}\right\},
$$

with $C \subset \operatorname{Cen}\left(M_{J}\right)$ and $C^{\prime} \subset \operatorname{Cen}\left(M_{J^{\prime}}\right)$ being closed subgroups and $\theta_{c}: M_{J} / C \rightarrow M_{J^{\prime}} / C^{\prime}$ a group isomorphism mapping $H / \operatorname{Cen}\left(M_{J}\right)$ to $H / \operatorname{Cen}\left(M_{J^{\prime}}\right)$ and $U^{\alpha}$ to $U^{c(\alpha)}$ for every $\alpha \in J$. For an admissible quadruple $\mathcal{C}=\left(J, J^{\prime}, c, L\right)$, let

$$
R_{\mathcal{C}}=L\left(U_{J} \times U_{J^{\prime}}\right) \subset P_{J} \times P_{J^{\prime}}
$$


For example, $R_{\mathcal{C}}=B \times B$ for $\mathcal{C}=(\emptyset, \emptyset$, Id, $H \times H)$ and $R_{\mathcal{C}}=G_{\text {diag }}$ for $\mathcal{C}=\left(\Gamma, \Gamma, \mathrm{Id}, G_{\text {diag }}\right)$. For an admissible quadruple $\mathcal{C}=\left(J, J^{\prime}, c, L\right)$, let

$$
Z_{\mathcal{C}}=(G \times G) / R_{\mathcal{C}}
$$

When $G$ is of adjoint type, the $G \times G$-orbits in the De Concini-Procesi compactification $\bar{G}$ of $G$ are all of the form $Z_{\mathcal{C}}$ for some admissible quadruples $\mathcal{C}$ (see $\$ 3.1$ ).

2.2. For $(x, y) \in W^{J} \times W$, let

$$
\begin{aligned}
& {[\mathcal{C}, x, y]=(B \times B)(x, y) \cdot R_{\mathcal{C}} \subset Z_{\mathcal{C}}} \\
& {[\mathcal{C}, x, y]^{-,+}=\left(B^{-} \times B\right)(x, y) \cdot R_{\mathcal{C}} \subset Z_{\mathcal{C}}} \\
& {[\mathcal{C}, x, y]^{-,-}=\left(B^{-} \times B^{-}\right)(x, y) \cdot R_{\mathcal{C}} \subset Z_{\mathcal{C}}}
\end{aligned}
$$

It follows from [27] that

$$
Z_{\mathcal{C}}=\bigsqcup_{(x, y) \in W^{J} \times W}[\mathcal{C}, x, y]=\bigsqcup_{(x, y) \in W^{J} \times W}[\mathcal{C}, x, y]^{-,+}=\bigsqcup_{(x, y) \in W^{J} \times W}[\mathcal{C}, x, y]^{-,-}
$$

are the partitions of $Z_{\mathcal{C}}$ by the $B \times B, B^{-} \times B$, and $B^{-} \times B^{-}$-orbits, respectively.

2.3. Let $\delta$ be an automorphism of $G$ preserving both $H$ and $B$, and let

$$
G_{\delta}=\{(g, \delta(g)): g \in G\} \subset G \times G
$$

be the graph of $\delta$. For $w \in W^{J}$, let

$$
Z_{\mathcal{C}, \delta, w}=G_{\delta}(B \times B)(w, 1) \cdot R_{\mathcal{C}} \subset Z_{\mathcal{C}} .
$$

The sets $Z_{\mathcal{C}, \delta, w}$ for $w \in W^{J}$ will be called the $G_{\delta}$-stable pieces in $Z_{\mathcal{C}}$. By [12, 20, 28], each $Z_{\mathcal{C}, \delta, w}$ is a locally closed smooth irreducible subset of $Z_{\mathcal{C}}$, and

$$
Z_{\mathcal{C}}=\bigsqcup_{w \in W^{J}} Z_{\mathcal{C}, \delta, w}
$$

is the partition of $Z_{\mathcal{C}}$ by the $G_{\delta^{-}}$stable pieces.

2.4. We now recall the closure relations of the $B \times B$-orbits and $G_{\delta^{-}}$ stable pieces in $Z_{\mathcal{C}}$. For $X \subset Z_{\mathcal{C}}$, let $\bar{X}$ be the closure of $X$ in $Z_{\mathcal{C}}$.

1) For $(x, y) \in W^{J} \times W, \overline{[\mathcal{C}, x, y]}=\bigsqcup\left[\mathcal{C}, x^{\prime}, y^{\prime}\right]$, where $\left(x^{\prime}, y^{\prime}\right)$ runs over elements in $W^{J} \times W$ such that $x^{\prime} u \leqslant x$ and $y^{\prime} c(u) \leqslant y$ for some $u \in W_{J}$. See [20, Corollary 4.1].

2) For $w \in W^{J}, \overline{Z_{\mathcal{C}, \delta, w}}=\bigsqcup Z_{\mathcal{C}, \delta, w^{\prime}}$, where $w^{\prime}$ runs over elements in $W^{J}$ such that $\delta^{-1}(c(u)) w^{\prime} u^{-1} \leqslant w$ for some $u \in W_{J}$. See [13, Corollary 5.9].

Using that facts that

$$
\begin{aligned}
& {[\mathcal{C}, x, y]^{-,+}=\left(w_{0}, 1\right)\left[\mathcal{C}, w_{0} x w_{0}^{J}, y w_{0}^{J^{\prime}}\right]} \\
& {[\mathcal{C}, x, y]^{-,-}=\left(w_{0}, w_{0}\right)\left[\mathcal{C}, w_{0} x w_{0}^{J}, w_{0} y w_{0}^{J^{\prime}}\right],}
\end{aligned}
$$

one has the following variations of 1 ). 
3) For $(x, y) \in W^{J} \times W, \overline{[\mathcal{C}, x, y]^{-,+}}=\bigsqcup\left[\mathcal{C}, x^{\prime}, y^{\prime}\right]^{-,+}$, where $\left(x^{\prime}, y^{\prime}\right)$ runs over elements in $W^{J} \times W$ such that $x^{\prime} u \geqslant x w_{0}^{J}$ and $y^{\prime} c(u) \leqslant y w_{0}^{J^{\prime}}$ for some $u \in W_{J}$.

4) For $(x, y) \in W^{J} \times W, \overline{[\mathcal{C}, x, y]^{-,-}}=\bigsqcup\left[\mathcal{C}, x^{\prime}, y^{\prime}\right]^{-,-}$, where $\left(x^{\prime}, y^{\prime}\right)$ runs over elements in $W^{J} \times W$ such that $x^{\prime} u \geqslant x w_{0}^{J}$ and $y^{\prime} c(u) \geqslant y w_{0}^{J^{\prime}}$ for some $u \in W_{J}$.

2.5. Recall that the monoid operation $*$ on $W$ is defined by $\overline{B(x * y) B}=$ $\overline{B x B y B}$ for $x, y \in W$. Similarly, for $x, y \in W$, define $x \triangleright y \in W$ and $x \triangleleft y \in W$ by

$$
\overline{B x B y B^{-}}=\overline{B(x \triangleright y) B^{-}} \text {and } \overline{B^{-} x B y B}=\overline{B^{-}(x \triangleleft y) B} .
$$

Then

$$
(W, *) \times W \longrightarrow W:(x, y) \longmapsto x \triangleright y, \quad x, y \in W
$$

is a left monoid action of $(W, *)$ on $W$, and

$$
W \times(W, *) \longrightarrow W:(x, y) \longmapsto x \triangleleft y, \quad x, y \in W
$$

is a right monoidal action of $(W, *)$ on $W$. More properties of $*, \triangleright$ and $\triangleleft$ are reviewed in the Appendix.

2.6. We now determine when the intersection of a $B \times B$-orbit and a $B^{-} \times B^{-}$-orbit in $Z_{\mathcal{C}}$ is non-empty.

Proposition 2.1. For any $(x, y),(u, v) \in W^{J} \times W$, the following conditions are equivalent:

1) $[\mathcal{C}, x, y] \cap[\mathcal{C}, u, v]^{-,-} \neq \emptyset$,

2) $u \leqslant x$ and $\min \left(v W_{J^{\prime}}\right) \leqslant y$,

3) $u \leqslant x$ and $v \leqslant \max \left(y W_{J^{\prime}}\right)$.

Proof. Using the facts that $x, u \in W^{J}$, it is easy to see that

$$
\begin{aligned}
& {[\mathcal{C}, x, y]=(B \times B)(x, y)(B \times B) \cdot R_{\mathcal{C}}} \\
& {[\mathcal{C}, u, v]^{-,-}=\left(B^{-} \times B^{-}\right)\left(u w_{0}^{J}, v w_{0}^{J^{\prime}}\right)(B \times B) \cdot R_{\mathcal{C}} .}
\end{aligned}
$$

Thus $[\mathcal{C}, x, y] \cap[\mathcal{C}, u, v]^{-,-} \neq \emptyset$ if and only if

$$
(B x B, B y B) \cap\left(\left(B^{-} \times B^{-}\right)\left(u w_{0}^{J}, v w_{0}^{J^{\prime}}\right)(B \times B) R_{\mathcal{C}}(B \times B)\right) \neq \emptyset .
$$

Since

$$
(B \times B) R_{\mathcal{C}}(B \times B)=\bigcup_{z \in W_{J}}(B \times B)(z, c(z))(B \times B),
$$

$[\mathcal{C}, x, y] \cap[\mathcal{C}, u, v]^{-,-} \neq \emptyset$ if and only if

$$
(B x B, B y B) \cap\left(B^{-} u w_{0}^{J} B z B, B^{-} v w_{0}^{J^{\prime}} B c(z) B\right) \neq \emptyset
$$

for some $z \in W_{J}$. By Lemma 5.7 and Lemma 5.3 in the Appendix, (5) is the same as

$$
u w_{0}^{J} \leqslant x * z^{-1} \quad \text { and } \quad v w_{0}^{J^{\prime}} \leqslant y * c(z)^{-1} \quad \text { for some } z \in W_{J} .
$$


Since for any $z \in W_{J}, x * z \leqslant \max \left(x W_{J}\right)$ and $y * c(z) \leqslant \max \left(y W_{J^{\prime}}\right)$ and both inequalities become equalities when $z=w_{0}^{J}$, (5) is equivalent to $u w_{0}^{J} \leqslant \max \left(x W_{J}\right)$ and $v w_{0}^{J^{\prime}} \leqslant \max \left(y W_{J^{\prime}}\right)$ which, by Lemma 5.5 in the Appendix, are in turn equivalent to $u \leqslant x$ and $\min \left(v W_{J^{\prime}}\right) \leqslant y$, or $u \leqslant x$ and $v \leqslant \max \left(y W_{J^{\prime}}\right)$.

Example 2.1. When $R_{\mathcal{C}}=G_{\text {diag }}$ and $Z_{\mathcal{C}}$ is identified with $G$, the intersections in Proposition 2.1 are of the form $B y B \cap B^{-} w B^{-}$for $y, w \in W$, and are called double Bruhat cells [8]. It is well-known (see, for example, [8]) that the intersection $(B y B) \cap\left(B^{-} w B^{-}\right)$is non-empty for all $y, w \in W$, which can also be seen from Proposition 2.1.

2.7. We now determine when the intersection of a $G_{\delta}$-stable piece and a $B^{-} \times B$-orbit in $Z_{\mathcal{C}}$ is nonempty.

Proposition 2.2. For $w \in W^{J}$ and $(x, y) \in W^{J} \times W$, the following conditions are equivalent:

1) $Z_{\mathcal{C}, \delta, w} \cap[\mathcal{C}, x, y]^{-,+} \neq \emptyset$

2) $y^{-1} \triangleright \delta(x) \leqslant \max \left(W_{J^{\prime}} \delta(w)\right)$,

3) $\min \left(W_{J^{\prime}}\left(y^{-1} \triangleright \delta(x)\right)\right) \leqslant \delta(w)$.

Proof. Using the facts that $w, x \in W^{J}$, it is easy to see that

$$
\begin{aligned}
& Z_{\mathcal{C}, \delta, w}=G_{\delta}(B \times B)(w, 1)(B \times B) \cdot R_{\mathcal{C}} \\
& {[\mathcal{C}, x, y]^{-,+}=\left(B^{-} \times B\right)\left(x w_{0}^{J}, y w_{0}^{J^{\prime}}\right)(B \times B) \cdot R_{\mathcal{C}} .}
\end{aligned}
$$

Thus $Z_{\mathcal{C}, \delta, w} \cap[\mathcal{C}, x, y]^{-,+} \neq \emptyset$ if and only if $G_{\delta} \cap\left(\left(B^{-} \times B\right)\left(x w_{0}^{J}, y w_{0}^{J^{\prime}}\right)(B \times B) R_{\mathcal{C}}(B \times B)\left(w^{-1}, 1\right)(B \times B)\right) \neq \emptyset$.

By (44), $Z_{\mathcal{C}, \delta, w} \cap[\mathcal{C}, x, y]^{-,+} \neq \emptyset$ if and only if

$G_{\delta} \cap\left(\left(B^{-} \times B\right)\left(x w_{0}^{J}, y w_{0}^{J^{\prime}}\right)(B \times B)(z, c(z))(B \times B)\left(w^{-1}, 1\right)(B \times B)\right) \neq \emptyset$

for some $z \in W_{J}$, which is equivalent to

$$
\left(B^{-} \delta\left(x w_{0}^{J}\right) B \delta(z) B \delta\left(w^{-1}\right) B\right) \cap\left(B y w_{0}^{J^{\prime}} B c(z) B\right) \neq \emptyset .
$$

Since $l\left(z w^{-1}\right)=l(z)+l\left(w^{-1}\right)$ for every $z \in W_{J}$, (6) is equivalent to

$$
\left(B^{-} \delta\left(x w_{0}^{J}\right) B \delta\left(z w^{-1}\right) B\right) \cap\left(B y w_{0}^{J^{\prime}} B c(z) B\right) \neq \emptyset
$$

which, by Lemma 5.7 and Lemma 5.3 in the Appendix, is equivalent to

$$
\delta\left(x w_{0}^{J}\right) \leqslant\left(y w_{0}^{J^{\prime}}\right) * c(z) * \delta(w) * \delta\left(z^{-1}\right)
$$

for some $z \in W_{J}$. Since by Lemma 5.4 in the Appendix,

$$
\begin{aligned}
\left(y w_{0}^{J^{\prime}}\right) * c(z) * \delta(w) * \delta\left(z^{-1}\right) & \leqslant\left(y w_{0}^{J^{\prime}}\right) * c\left(w_{0}^{J}\right) * \delta(w) * \delta\left(w_{0}^{J}\right) \\
& =y * \max \left(W_{J^{\prime}} \delta(w) W_{\delta(J)}\right)
\end{aligned}
$$


for any $z \in W_{J}$ with equality holds when $z=w_{0}^{J}$, (7) is equivalent to

$$
\delta\left(x w_{0}^{J}\right) \leqslant y * \max \left(W_{J^{\prime}} \delta(w) W_{\delta(J)}\right) .
$$

Clearly (8) leads to

$$
\delta(x) \leqslant y * \max \left(W_{J^{\prime}} \delta(w) W_{\delta(J)}\right) .
$$

Conversely, if (9) holds, then

$$
\begin{aligned}
\delta\left(x w_{0}^{J}\right) & =\delta(x) * w_{0}^{\delta(J)} \leqslant y * \max \left(W_{J^{\prime}} \delta(w) W_{\delta(J)}\right) * w_{0}^{\delta(J)} \\
& =y * \max \left(W_{J^{\prime}} \delta(w) W_{\delta(J)}\right) .
\end{aligned}
$$

Thus (81) is equivalent to (9), which, by Lemma 5.3 in the Appendix, is equivalent to

$$
y^{-1} \triangleright \delta(x) \leqslant \max \left(W_{J^{\prime}} \delta(w) W_{\delta(J)}\right) .
$$

Since $y^{-1} \triangleright \delta(x) \in W^{\delta(J)}$ by Lemma 5.4 in the Appendix, and since

$$
\max \left(W_{J^{\prime}} \delta(w) W_{\delta(J)}\right)=\max \left(W_{J^{\prime}} \delta(w)\right) * w_{0}^{\delta(J)}
$$

(10) is equivalent to $y^{-1} \triangleright \delta(x) \leqslant \max \left(W_{J^{\prime}} \delta(w)\right)$ by Lemma 5.5 in the Appendix. The equivalence of 2) and 3) also follows from Lemma 5.5 in the Appendix.

2.8. We now discuss some consequences of the results in $\$ 2.6$ and $\$ 2.7$.

Corollary 2.1. Let $J \subset \Gamma$. For $(x, y) \in W^{J} \times W$. Set

$$
w_{x, y}=\min \left(W_{\delta^{-1}\left(J^{\prime}\right)}\left(\delta^{-1}\left(y^{-1}\right) \triangleright x\right)\right) \in{ }^{\delta^{-1}\left(J^{\prime}\right)} W^{J} .
$$

Then for $w \in W^{J}$,

$$
Z_{\mathcal{C}, \delta, w} \cap[\mathcal{C}, x, y]^{-,+} \neq \emptyset \quad \text { iff } \quad Z_{\mathcal{C}, \delta, w_{x, y}} \subset \overline{Z_{\mathcal{C}, \delta, w}} .
$$

Proof. If $Z_{\mathcal{C}, \delta, w} \cap[\mathcal{C}, x, y]^{-,+} \neq \emptyset$, by Proposition [2.2, $w_{x, y} \leqslant w$. By $\oint 2.4$, 2), $Z_{\mathcal{C}, \delta, w_{x, y}} \subset \overline{Z_{\mathcal{C}, \delta, w}}$. On the other hand, if $Z_{\mathcal{C}, \delta, w_{x, y}} \subset \overline{Z_{\mathcal{C}, \delta, w}}$, then there exists $u \in W_{J}$ such that $\delta^{-1}(c(u)) w_{x, y} u^{-1} \leqslant w$. Since $w_{x, y} \in \delta^{-1}\left(J^{\prime}\right) W^{J}, w_{x, y} \leqslant \delta^{-1}(c(u)) w_{x, y} u^{-1} \leqslant w$. By Proposition 2.2, $Z_{\mathcal{C}, \delta, w} \cap[\mathcal{C}, x, y]^{-,+} \neq \emptyset$.

Proposition 2.3. Let $\pi: Z_{\mathcal{C}} \rightarrow(G \times G) /\left(P_{J} \times P_{J^{\prime}}\right)$ be the natural projection induced by the inclusion $R_{\mathcal{C}} \subset P_{J} \times P_{J^{\prime}}$. Then for any $w \in W^{J}$ and $(x, y) \in W^{J} \times W$,

$$
Z_{\mathcal{C}, \delta, w} \cap[\mathcal{C}, x, y]^{-,+} \neq \emptyset \quad \text { iff } \quad \pi\left(Z_{\mathcal{C}, \delta, w}\right) \cap \pi\left([\mathcal{C}, x, y]^{-,+}\right) \neq \emptyset .
$$

Proof. Clearly $Z_{\mathcal{C}, \delta, w} \cap[\mathcal{C}, x, y]^{-,+} \neq \emptyset$ implies that $\pi\left(Z_{\mathcal{C}, \delta, w}\right) \cap$ $\pi\left([\mathcal{C}, x, y]^{-,+}\right) \neq \emptyset$. Assume now that $\pi\left(Z_{\mathcal{C}, \delta, w}\right) \cap \pi\left([\mathcal{C}, x, y]^{-,+}\right) \neq \emptyset$. Let $y^{\prime}=\min \left(y W_{J^{\prime}}\right) \in W^{J^{\prime}}$ and $w^{\prime}=\min \left(W_{\delta^{-1}\left(J^{\prime}\right)} w\right) \in{ }^{\delta^{-1}\left(J^{\prime}\right)} W^{J}$. Then

$$
\begin{aligned}
& \pi\left(Z_{\mathcal{C}, \delta, w}\right)=G_{\delta}\left(w^{\prime}, 1\right)\left(P_{J} \times P_{J^{\prime}}\right) \\
& \pi\left([\mathcal{C}, x, y]^{-,+}\right)=\left(B^{-} \times B\right)\left(x, y^{\prime}\right)\left(P_{J} \times P_{J^{\prime}}\right) .
\end{aligned}
$$


By definition, $\max \left(W_{J^{\prime}} \delta(w)\right)=\max \left(W_{J^{\prime}} \delta\left(w^{\prime}\right)\right)$. By Lemma 5.2 in the Appendix, $y^{-1} \triangleright \delta(x) \leqslant\left(y^{\prime}\right)^{-1} \triangleright \delta(x)$. Now $Z_{\mathcal{C}, \delta, w} \cap[\mathcal{C}, x, y]^{-,+} \neq \emptyset$ follows from Proposition 2.2 and the following Lemma 2.1.

Lemma 2.1. For $w \in \delta^{\delta^{-1}\left(J^{\prime}\right)} W^{J}$ and $(x, y) \in W^{J} \times W^{J^{\prime}}$,

$$
G_{\delta}(w, 1)\left(P_{J} \times P_{J^{\prime}}\right) \cap\left(B^{-} \times B\right)(x, y)\left(P_{J} \times P_{J^{\prime}}\right) \neq \emptyset
$$

if and only if $y^{-1} \triangleright \delta(x) \leqslant \max \left(W_{J^{\prime}} \delta(w)\right)$.

Proof. First note that

$$
G_{\delta}(w, 1)\left(P_{J} \times P_{J^{\prime}}\right)=G_{\delta}(B \times B)(w, 1)\left(P_{J} \times P_{J^{\prime}}\right) .
$$

Thus $G_{\delta}(w, 1)\left(P_{J} \times P_{J^{\prime}}\right) \cap\left(B^{-} \times B\right)(x, y)\left(P_{J} \times P_{J^{\prime}}\right) \neq \emptyset$ if and only if

$$
G_{\delta} \cap\left(B^{-} \times B\right)(x, y)\left(P_{J} \times P_{J^{\prime}}\right)\left(w^{-1}, 1\right)(B \times B) \neq \emptyset .
$$

Using $P_{J} \times P_{J^{\prime}}=\cup_{z \in W_{J}, z^{\prime} \in W_{J^{\prime}}}(B \times B)\left(z, z^{\prime}\right)(B \times B)$ and the fact that $B z B w^{-1} B=B z w^{-1} B$ for any $z \in W_{J}$, one sees that (11) is equivalent to

$$
\bigcup_{z \in W_{J}, z^{\prime} \in W_{J^{\prime}}} G_{\delta} \cap\left(B^{-} x B z w^{-1} B \times B y B z^{\prime} B\right) \neq \emptyset,
$$

or $\left(B^{-} \delta(x) B \delta\left(z w^{-1}\right) B\right) \cap\left(B y B z^{\prime} B\right) \neq \emptyset$ for some $z \in W_{J}$ and $z^{\prime} \in W_{J^{\prime}}$, which, by Lemma 5.7 and Lemma 5.3 in the Appendix, is in turn equivalent to

$$
y^{-1} \triangleright \delta(x) \leqslant z^{\prime} * \delta(w) * \delta\left(z^{-1}\right) \quad \text { for some } z \in W_{J}, z^{\prime} \in W_{J^{\prime}} .
$$

Since for any $z \in W_{J}$ and $z^{\prime} \in W_{J^{\prime}}$,

$$
z^{\prime} * \delta(w) * \delta\left(z^{-1}\right) \leqslant w_{0}^{J^{\prime}} * \delta(w) * w_{0}^{\delta(J)}=\max \left(W_{J^{\prime}} \delta(w) W_{\delta(J)}\right)
$$

with equality holds when $z=w_{0}^{J}$ and $z^{\prime}=w_{0}^{J^{\prime}}$, (11) is equivalent to

$$
y^{-1} \triangleright \delta(x) \leqslant \max \left(W_{J^{\prime}} \delta(w) W_{\delta(J)}\right) .
$$

Since $\delta(x) \in W^{\delta(J)}$, it follows from Lemma 5.4 in the Appendix that (12) is equivalent to $y^{-1} \triangleright \delta(x) \leqslant \max \left(W_{J^{\prime}} \delta(w)\right)$.

2.9. To study the geometry and closures of the non-empty intersections in $\$ 2.6$ and $\$ 2.7$, we first recall some elementary facts on intersections of subvarieties in an algebraic variety.

The following Lemma 2.2 is a generalization of [24, Corollary 1.5] of Richardson. Our proof of Lemma 2.2 is essentially the same as that of [24, Theorem 1.4].

Lemma 2.2. Let $A$ be a connected algebraic group and let $H, K$ and $L$ be closed connected subgroups of $A$. Assume that $H \cap K$ is connected and that $\operatorname{Lie}(H)+\operatorname{Lie}(K)=\operatorname{Lie}(A)$. Let $Y$ be an irreducible subvariety of $A / L$ such that $H Y \subset A / L$ is smooth. Then for any $K$-orbit $O$ in $A / L$ such that $(H Y) \cap O \neq \emptyset, H Y$ and $O$ intersect transversally in $A / L$ and $H Y \cap O$ is a smooth irreducible subvariety of $A / L$ with

$$
\operatorname{dim}((H Y) \cap O)=\operatorname{dim} H Y+\operatorname{dim} O-\operatorname{dim} A / L .
$$


Proof. Since $H Y$ is a union of $H$-orbits in $A / L$, it follows from 24, Corollary 1.5] and [24, Proposition 1.2] that $H Y$ and $O$ intersect transversally and that the intersection $(H Y) \cap O$ is smooth. Moreover, each irreducible component of $(H Y) \cap O$ has dimensional equal to $\operatorname{dim} H Y+\operatorname{dim} O-\operatorname{dim} A / L$.

It remains to show that $(H Y) \cap O$ is irreducible. Fix an $x \in O$ and consider the diagram

$$
O \stackrel{p}{\longleftarrow} H \times K \stackrel{m}{\longrightarrow} A \stackrel{q}{\longrightarrow} A / L,
$$

where $p(h, k)=k x, m(h, k)=h^{-1} k$, and $q(a)=a x$ for $h \in H, k \in K$, and $a \in A$. Let

$$
E=\left\{(h, k) \in H \times K: h^{-1} k x \in Y\right\} \subset H \times K .
$$

Then $(H Y) \cap O=p(E)$, so it is enough to show that $E$ is irreducible.

Since $L$ is connected and $Y \subset A / L$ is irreducible, $q^{-1}(Y) \subset A$ is irreducible by [24, Lemma 1.3]. As in the proof of [24, Theorem 1.4], $H K$ is open in $A$, so $H K \cap q^{-1}(Y)$ is an irreducible subvariety of $H K$. The map $m$ induces an isomorphism $m:(H \times K) / D \rightarrow H K$, where $D=\{(g, g): g \in H \cap K\}$. Let $\nu: H \times K \rightarrow(H \times K) / D$ be the natural projection. Since $D$ is connected, by [24, Lemma 1.3], $E=\nu^{-1}\left(m^{-1}\left(H K \cap q^{-1}(Y)\right)\right)$ is also irreducible.

The following Lemma 2.3 is useful in determining the irreducible components of intersections of algebraic varieties and will be used several times in the paper.

Lemma 2.3. Let $Y$ be an algebraic variety over an algebraically closed field $\mathbf{k}$. Suppose that $l \geq 0$ is an integer such that every irreducible component of $Y$ has dimension at least $l$, and suppose that $Y=\bigsqcup_{k \in \mathcal{K}} Y_{k}$ is a finite disjoint union, where each $Y_{k}$ is an irreducible subvariety of $Y$ with $\operatorname{dim} Y_{k} \leqslant l$. Then the irreducible components of $Y$ are precisely the closures $\overline{Y_{k}}$ of those $Y_{k}$ 's, where $k \in \mathcal{K}$ and $\operatorname{dim} Y_{k}=l$.

Proof. Let $S$ be any irreducible component of $Y$. Then

$$
S=\bigcup_{k \in \mathcal{K}: S \cap Y_{k} \neq \emptyset} \overline{S \cap Y_{k}}
$$

Since $S$ is irreducible, $S=\overline{S \cap Y_{k}} \subset \overline{Y_{k}}$ for some $k \in \mathcal{K}$. Since $\overline{Y_{k}}$ is irreducible, $S=\overline{Y_{k}}$, and it follows from the dimension assumptions that $\operatorname{dim} Y_{k}=l$. Since the $Y_{k}$ 's are pair-wise disjoint, the closures $\overline{Y_{k}}$ with $\operatorname{dim} Y_{k}=l$ are pair-wise distinct irreducible components.

Lemma 2.4. Let $Z$ be a smooth irreducible algebraic variety and let

$$
Z=\bigsqcup_{i \in \mathcal{I}} X_{i}=\bigsqcup_{j \in \mathcal{J}} Y_{j}
$$


be two partitions of $Z$ such that each non-empty intersection $X_{i} \cap Y_{j}$ is transversal and irreducible. Then for any $(i, j) \in \mathcal{I} \times \mathcal{J}, X_{i} \cap Y_{j} \neq \emptyset$ if and only if $\overline{X_{i}} \cap \overline{Y_{j}} \neq \emptyset$, and in this case,

$$
\overline{X_{i} \cap Y_{j}}=\overline{X_{i}} \cap \overline{Y_{j}} \text {. }
$$

In particular, $Z=\bigsqcup_{(i, j) \in \mathcal{K}}\left(X_{i} \cap Y_{j}\right)$ is again a partition of $Z$. Here $\mathcal{K}=\left\{(i, j) \in \mathcal{I} \times \mathcal{J}: X_{i} \cap Y_{j} \neq \emptyset\right\}$.

Proof. Let $(i, j) \in \mathcal{I} \times \mathcal{J}$ be such that $\overline{X_{i}} \cap \overline{Y_{j}} \neq \emptyset$, and let

$$
\mathcal{K}_{i j}=\left\{\left(i^{\prime}, j^{\prime}\right) \in \mathcal{I} \times \mathcal{J}: X_{i^{\prime}} \subset \overline{X_{i}}, Y_{j^{\prime}} \subset \overline{Y_{j}}, X_{i^{\prime}} \cap Y_{j^{\prime}} \neq \emptyset\right\} .
$$

Then

$$
\overline{X_{i}} \cap \overline{Y_{j}}=\bigsqcup_{\left(i^{\prime}, j^{\prime}\right) \in \mathcal{K}_{i j}} X_{i^{\prime}} \cap Y_{j^{\prime}}
$$

is a disjoint union. By [10, Page 222], every irreducible component of $\overline{X_{i}} \cap \overline{Y_{j}}$ has dimension at least $\operatorname{dim} X_{i}+\operatorname{dim} Y_{j}-\operatorname{dim} Z$. On the other hand, for any $\left(i^{\prime}, j^{\prime}\right) \in \mathcal{K}_{i j}$,

$$
\begin{aligned}
\operatorname{dim} X_{i^{\prime}} \cap Y_{j^{\prime}} & =\operatorname{dim} X_{i^{\prime}}+\operatorname{dim} Y_{j^{\prime}}-\operatorname{dim} Z \\
& \leqslant \operatorname{dim} X_{i}+\operatorname{dim} Y_{j}-\operatorname{dim} Z .
\end{aligned}
$$

Since $\overline{X_{i}}$ is irreducible, $\operatorname{dim} X_{i^{\prime}}<\operatorname{dim} X_{i}$ for any $i^{\prime} \in \mathcal{I}$ such that $X_{i^{\prime}} \subset \overline{X_{i}}$ and $i^{\prime} \neq i$. Similarly, $\operatorname{dim} Y_{j^{\prime}}<\operatorname{dim} Y_{j}$ for any $j^{\prime} \in \mathcal{J}$ such that $Y_{j^{\prime}} \subset \overline{Y_{j}}$ and $j^{\prime} \neq j$. Thus the inequality in (13) is an equality if and only if $\left(i^{\prime}, j^{\prime}\right)=(i, j)$. By Lemma 2.3, $X_{i} \cap Y_{j} \neq \emptyset$ and $\overline{X_{i} \cap Y_{j}}=\overline{X_{i}} \cap \overline{Y_{j}}$.

Theorem 2.1. Let $w \in W^{J}$ and $(x, y),(u, v) \in W^{J} \times W$. Then

1) $Z_{\mathcal{C}, \delta, w} \cap[\mathcal{C}, x, y]^{-,+} \neq \emptyset$ if and only if $\overline{Z_{\mathcal{C}, \delta, w}} \cap \overline{[\mathcal{C}, x, y]^{-,+}} \neq \emptyset$. In this case, $Z_{\mathcal{C}, \delta, w}$ and $[\mathcal{C}, x, y]^{-,+}$intersects transversally in $Z_{\mathcal{C}}$, the intersection is smooth and irreducible, and

$$
\overline{Z_{\mathcal{C}, \delta, w} \cap[\mathcal{C}, x, y]^{-,+}}=\overline{Z_{\mathcal{C}, \delta, w}} \cap \overline{[\mathcal{C}, x, y]^{-,+}} .
$$

2) $[\mathcal{C}, x, y] \cap[\mathcal{C}, u, v]^{-,-} \neq \emptyset$ if and only if $\overline{[\mathcal{C}, x, y]} \cap \overline{[\mathcal{C}, u, v]^{-,-}} \neq \emptyset$. In this case, $[\mathcal{C}, x, y]$ and $[\mathcal{C}, u, v]^{-,-}$intersects transversally in $Z_{\mathcal{C}}$, the intersection is smooth and irreducible, and

$$
\overline{[\mathcal{C}, x, y] \cap[\mathcal{C}, u, v]^{-,-}}=\overline{[\mathcal{C}, x, y]} \cap \overline{[\mathcal{C}, u, v]^{-,-}} .
$$

Proof. Since $R_{\mathcal{C}}$ is connected, $G_{\delta} \cap\left(B^{-} \times B\right)$ is connected, and

$$
\operatorname{Lie}\left(G_{\delta}\right)+\operatorname{Lie}\left(B^{-} \times B\right)=\operatorname{Lie}(G \times G),
$$

Lemma 2.2 applies. By taking $A=G \times G$,

$$
H=G_{\delta}, \quad K=B^{-} \times B, \quad L=R_{\mathcal{C}}, \text { and } Y=(B \times B)(w, 1) \cdot R_{\mathcal{C}}
$$

in Lemma 2.2 , one sees that when $Z_{\mathcal{C}, \delta, w} \cap[\mathcal{C}, x, y]^{-,+} \neq \emptyset, Z_{\mathcal{C}, \delta, w}$ and $[\mathcal{C}, x, y]^{-,+}$intersect transversally in $Z_{\mathcal{C}}$, and that the intersection 
$Z_{\mathcal{C}, \delta, w} \cap[\mathcal{C}, x, y]^{-,+}$is smooth and irreducible. By applying Lemma 2.4 to the two partitions

$$
Z_{\mathcal{C}}=\bigsqcup_{w \in W^{J}} Z_{\mathcal{C}, \delta, w}=\bigsqcup_{(x, y) \in W^{J} \times W}\left(B^{-} \times B\right)(x, y) \cdot R_{\mathcal{C}}
$$

of $Z_{\mathcal{C}}$, one proves part 1). Part 2) can be proved in the same way.

Remark 2.1. In both 1) and 2) in Theorem 2.1, the fact that the intersection is non-empty if and only if the intersection of the closures is non-empty can also been obtained using \$2.4 and Proposition 2.1 and Proposition 2.2, However, the proof we give is more conceptual.

\section{INTERSECTIONS IN $\bar{G}$}

3.1. Let $G$ be a connected semi-simple adjoint group and $\bar{G}$ be the De Concini-Procesi compactification. It is well-known that the $G \times G$ orbits in $\bar{G}$ are in one to one correspondence with subsets of $\Gamma$. For $J \subset \Gamma$, let $Z_{J}$ be the corresponding $G \times G$-orbit in $\bar{G}$. A distinguished point $h_{J} \in Z_{J}$ can be chosen such that the stabilizer subgroup of $G \times G$ at $h_{J}$ is

$$
R_{J}^{-\stackrel{\text { def }}{=}}\left(U_{J}^{-} \times U_{J}\right)\left\{\left(m_{1}, m_{2}\right) \in M_{J} \times M_{J}: \pi_{J}\left(m_{1}\right)=\pi_{J}\left(m_{2}\right)\right\},
$$

where $\pi_{J}: M_{J} \rightarrow M_{J} / \operatorname{Cen}\left(M_{J}\right)$ is the natural projection and $\operatorname{Cen}\left(M_{J}\right)$ is the center of $M_{J}$.

For $J \subset \Gamma$, let $\mathcal{C}_{J}=\left(J^{*}, J, c, L\right)$, where $J^{*}=-w_{0}(J), c=\left(w_{0} w_{0}^{J}\right)^{-1}$, and

$$
L=\left\{\left(\dot{w}_{0} \dot{w}_{0}^{J} m_{1}\left(\dot{w}_{0} \dot{w}_{0}^{J}\right)^{-1}, m_{2}\right): m_{1}, m_{2} \in M_{J}, \pi_{J}\left(m_{1}\right)=\pi_{J}\left(m_{2}\right)\right\}
$$

with $\dot{w}_{0}$ and $\dot{w}_{0}^{J}$ being any representatives of $w_{0}$ and $w_{0}^{J}$ in $N_{G}(H)$. By [20, Section 5], $\mathcal{C}_{J}$ is an admissible quadruple for $G$, and

$$
R_{\mathcal{C}_{J}}=\left(\dot{w}_{0} \dot{w}_{0}^{J}, 1\right) R_{J}^{-}\left(\dot{w}_{0} \dot{w}_{0}^{J}, 1\right)^{-1} .
$$

One thus has the isomorphism

$$
Z_{J} \longrightarrow Z_{\mathcal{C}_{J}}: \quad\left(g, g^{\prime}\right) \cdot h_{J} \longmapsto\left(g w_{0}^{J} w_{0}, g^{\prime}\right) \cdot R_{\mathcal{C}_{J}}, \quad g, g^{\prime} \in G .
$$

3.2. For $J \subset \Gamma$ and $(x, y) \in W^{J} \times W$, let

(15) $[J, x, y]=(B \times B)(x, y) \cdot h_{J}$,

(16) $[J, x, y]^{-,+}=\left(B^{-} \times B\right)(x, y) \cdot h_{J}=\left(w_{0}, 1\right)\left[J, w_{0} x w_{0}^{J}, y w_{0}^{J}\right]$,

(17) $[J, x, y]^{-,-}=\left(B^{-} \times B^{-}\right)(x, y) \cdot h_{J}=\left(w_{0}, w_{0}\right)\left[J, w_{0} x w_{0}^{J}, w_{0} y w_{0}^{J}\right]$.

For $J \subset \Gamma$ and $w \in W^{J}$, let

$$
Z_{J, \delta, w}=G_{\delta}(B \times B)(w, 1) \cdot h_{J} .
$$


The $Z_{J, \delta, w}$ 's will be called the $G_{\delta}$-stable pieces in $\bar{G}$. By [12, 27], one has the following partitions of $\bar{G}$ :

$$
\begin{aligned}
\bar{G} & =\bigsqcup_{J \subset \Gamma,(x, y) \in W^{J} \times W}[J, x, y]=\bigsqcup_{J \subset \Gamma,(x, y) \in W^{J} \times W}[J, x, y]^{-,-} \\
& =\bigsqcup_{J \subset \Gamma,(x, y) \in W^{J} \times W}[J, x, y]^{-,+}=\bigsqcup_{J \subset \Gamma, w \in W^{J}} Z_{J, \delta, w} .
\end{aligned}
$$

For a an irreducible subvariety $X \subset Z_{J}$, let $\operatorname{Codim}_{Z_{J}}(X)$ be the codimension of $X$ in $Z_{J}$. Let $l$ be the length function of $W$. One has, for any $J \subset \Gamma$ and $w \in W^{J}$,

1) $\operatorname{dim} Z_{J}=\operatorname{dim} G-\operatorname{dim} \operatorname{Cen}\left(M_{J}\right)=\operatorname{dim} G-|\Gamma|+|J|$. See [2].

2) $\operatorname{Codim}_{Z_{J}}[J, x, y]=l\left(w_{0}\right)+l(x)-l(y)$. See [27, Lemma 1.3].

3) $\operatorname{Codim}_{Z_{J}} Z_{J, \delta, w}=l(w)$. See [23, Section 8].

By (16) and (17), one also has

4) $\operatorname{Codim}_{Z_{J}}[J, x, y]^{-,+}=2 l\left(w_{0}\right)-l\left(x w_{0}^{J}\right)-l\left(y w_{0}^{J}\right)$.

5) $\operatorname{Codim}_{Z_{J}}[J, x, y]^{-,-}=l\left(w_{0}\right)-l\left(x w_{0}^{J}\right)+l\left(y w_{0}^{J}\right)$.

3.3. The closure of a $G \times G$-orbit is described in [2, 3] as follows.

1) For $J \subset \Gamma, \overline{Z_{J}}=\bigsqcup_{K \subset J} Z_{K}$ is a smooth subvariety of $\bar{G}$.

The closure of a $B \times B$-orbit is described in [27, Proposition 2.4], and the following simplified version in 2) is found in [16. Proposition 6.3] and [20, Example 1.3]. The following 3) and 4) are obtained using (16) and (17).

2) For $J \subset \Gamma$ and $(x, y) \in W^{J} \times W, \overline{[J, x, y]}=\bigsqcup\left[K, x^{\prime}, y^{\prime}\right]$, where $K \subset J,\left(x^{\prime}, y^{\prime}\right) \in W^{K} \times W$ and there exists $u \in W_{J}$ such that $x u \leqslant$ $x^{\prime}, y^{\prime} \leqslant y u$.

3) For $J \subset \Gamma$ and $(x, y) \in W^{J} \times W, \overline{[J, x, y]^{-,+}}=\bigsqcup\left[K, x^{\prime}, y^{\prime}\right]^{-,+}$, where $K \subset J,\left(x^{\prime}, y^{\prime}\right) \in W^{K} \times W$ and there exists $u \in W_{J}$ such that $x^{\prime} w_{0}^{K} \leqslant x u, y^{\prime} w_{0}^{K} \leqslant y u$.

4) For $J \subset \Gamma$ and $(x, y) \in W^{J} \times W, \overline{[J, x, y]^{-,-}}=\bigsqcup\left[K, x^{\prime}, y^{\prime}\right]^{-,-}$, where $K \subset J,\left(x^{\prime}, y^{\prime}\right) \in W^{K} \times W$ and there exists $u \in W_{J}$ such that $x^{\prime} w_{0}^{K} \leqslant x u, y^{\prime} w_{0}^{K} \geqslant y u$.

For $J \subset \Gamma$ and $w \in W$, let

$$
C_{J}(w)=\left\{\delta^{-1}(u) w u^{-1}: u \in W_{J}\right\},
$$

and denote by $\operatorname{Min}\left(C_{J}(w)\right)$ the set of minimal length elements in $C_{J}(w)$. The closure of a $G_{\delta}$-stable piece is described in [12, Sections 3 and 4] as follows:

5) For $J \subset \Gamma$ and $w \in W^{J}, \overline{Z_{J, \delta, w}}=\bigsqcup Z_{K, \delta, w^{\prime}}$, where $K \subset J, w^{\prime} \in$ $W^{K}$, and $w^{\prime} \geqslant w_{1}$ for some $w_{1} \in \operatorname{Min}\left(C_{J}(w)\right)$.

3.4. We can now prove our first main result in this paper. 
Proposition 3.1. For $J \subset \Gamma, w \in W^{J}$, and $(x, y),(u, v) \in W^{J} \times W$,

$$
\begin{aligned}
& {[J, x, y] \cap[J, u, v]^{-,-} \neq \emptyset \text { iff } \quad x \leqslant u, v \leqslant \max \left(y W_{J}\right),} \\
& Z_{J, \delta, w} \cap[J, x, y]^{-,+} \neq \emptyset \text { iff } \min \left(W_{J} \delta(w)\right) \leqslant y^{-1} * \delta(x) .
\end{aligned}
$$

Proof. Let $\mathcal{C}_{J}$ be as in $\$ 3.1$ and recall the isomorphism $Z_{J} \rightarrow Z_{\mathcal{C}_{J}}$ in (14). Since $W^{J^{*}}=W^{J} w_{0}^{J} w_{0}$, one has

$$
[J, x, y] \cap[J, u, v]^{-,-} \neq \emptyset \text { iff }\left[\mathcal{C}_{J}, x w_{0}^{J} w_{0}, y\right] \cap\left[\mathcal{C}_{J}, u w_{0}^{J} w_{0}, v\right]^{-,-} \neq \emptyset,
$$

which, by Proposition 2.1, is equivalent to $u w_{0}^{J} w_{0} \leqslant x w_{0}^{J} w_{0}$ and $v \leqslant$ $\max \left(y W_{J}\right)$. Note that $u w_{0}^{J} w_{0} \leqslant x w_{0}^{J} w_{0}$ if and only if $u w_{0}^{J} \geqslant x w_{0}^{J}$, which is equivalent to $u \geqslant x$ since $x, u \in W^{J}$. Thus (18) is proved.

Similarly, $Z_{J, \delta, w} \cap[J, x, y]^{-,+} \neq \emptyset$ iff $Z_{\mathcal{C}, \delta, w w_{0}^{J} w_{0}} \cap\left[\mathcal{C}, x w_{0}^{J} w_{0}, y\right]^{-,+} \neq \emptyset$, which, by Proposition 2.2, is equivalent to

$$
y^{-1} \triangleright\left(\delta(x) w_{0}^{\delta(J)} w_{0}\right) \leqslant \max \left(W_{J} \delta(w) w_{0}^{\delta(J)} w_{0}\right) .
$$

By Lemma 5.5 and Lemma 5.3 in the Appendix, (20) is equivalent to

$$
\min \left(W_{J} \delta(w) w_{0}^{\delta(J)}\right) \leqslant y^{-1} *\left(\delta(x) w_{0}^{\delta(J)}\right),
$$

which is in turn equivalent to $\min \left(W_{J} \delta(w)\right) \leqslant y^{-1} * \delta(x)$.

3.5. Admissible partitions of $\bar{G}$. In order to generalize Theorem 2.1 to $\bar{G}$, we will introduce the notion "admissible partitions" and discuss some of their properties.

Definition 3.1. A partition of $\bar{G}$ is said to be admissible if it is of the form

$$
\bar{G}=\bigsqcup_{J \subset \Gamma} \bigsqcup_{\alpha \in \mathcal{A}_{J}} X_{J, \alpha}
$$

where for each $J \subset \Gamma$ and $\alpha \in \mathcal{A}_{J}, X_{J, \alpha} \subset Z_{J}$ and

$$
\operatorname{Codim}_{Z_{K}} X_{K, \alpha^{\prime}} \geqslant \operatorname{Codim}_{Z_{J}} X_{J, \alpha}
$$

for every $K \subset J$ and $X_{K, \alpha^{\prime}} \subset \overline{X_{\alpha}} \cap Z_{K}$. An admissible partition is said to be strongly admissible if $\overline{X_{J, \alpha}} \cap Z_{K} \neq \emptyset$ for every $K \subset J$ and $\alpha \in \mathcal{A}_{J}$.

Note that the partition $\bar{G}=\bigsqcup_{J \subset \Gamma} Z_{J}$ is strongly admissible.

Proposition 3.2. The partitions

$$
\begin{aligned}
\bar{G} & =\bigsqcup_{J \subset \Gamma,(x, y) \in W^{J} \times W}[J, x, y]=\bigsqcup_{J \subset \Gamma,(x, y) \in W^{J} \times W}[J, x, y]^{-,-} \\
& =\bigsqcup_{J \subset \Gamma,(x, y) \in W^{J} \times W}[J, x, y]^{-,+}=\bigsqcup_{J \subset \Gamma, w \in W^{J}} Z_{J, \delta, w}
\end{aligned}
$$

are strongly admissible. 
Proof. Let $K \subset J \subset \Gamma$ and $(x, y) \in W^{J} \times W$. If $\left(x^{\prime}, y^{\prime}\right) \in W^{K} \times W$ is such that $\left[K, x^{\prime}, y^{\prime}\right] \subset \overline{[J, x, y]}$, one knows from $₫ 3.3$ that there exists $u \in W_{J}$ such that $x^{\prime} \geqslant x u$ and $y^{\prime} \leqslant y u$. Hence

$$
\begin{aligned}
\operatorname{Codim}_{Z_{K}}\left[K, x^{\prime}, y^{\prime}\right] & =l\left(w_{0}\right)+l\left(x^{\prime}\right)-l\left(y^{\prime}\right) \geqslant l\left(w_{0}\right)+l(x u)-l(y u) \\
& =l\left(w_{0}\right)+l(x)+l(u)-l(y u) \geqslant l\left(w_{0}\right)+l(x)-l(y) \\
& =\operatorname{Codim}_{Z_{J}}[J, x, y] .
\end{aligned}
$$

Regard $x$ as in $W^{K}$. By $\oint 3.3,[K, x, y] \subset \overline{[J, x, y]} \cap Z_{K}$. Thus the first partition in (22) is strongly admissible. The second and third partitions of $\bar{G}$ in (22), being the translations by $\left(w_{0}, 1\right)$ and by $\left(w_{0}, w_{0}\right)$ of the first one, are thus also strongly admissible.

Consider now the partition of $\bar{G}$ into the $G_{\delta^{-}}$-stable pieces. Let $K \subset$ $J \subset \Gamma$ and $w \in W^{J}$. If $w^{\prime} \in W^{K}$ is such that $Z_{K, \delta, w^{\prime}} \subset \overline{Z_{J, \delta, w}}$, one knows from $\$ 3.3$ that there exists $w_{1} \in \operatorname{Min}\left(C_{J}(w)\right)$ such that $w^{\prime} \geqslant w_{1}$. Hence

$$
\operatorname{Codim}_{Z_{K}}\left(Z_{K, \delta, w^{\prime}}\right)=l\left(w^{\prime}\right) \geqslant l\left(w_{1}\right)=l(w)=\operatorname{Codim}_{Z_{J}}\left(Z_{J, \delta, w}\right) .
$$

Regard $w$ as in $W^{K}$. By \$3.3, $Z_{K, \delta, w} \subset \overline{Z_{J, \delta, w}} \cap Z_{K}$. Thus the partition $\bar{G}=\bigsqcup_{J \subset \Gamma, w \in W^{J}} Z_{J, \delta, w}$ is strongly admissible.

Definition-Notation 3.1. Two admissible partitions

$$
\bar{G}=\bigsqcup_{J \subset \Gamma} \bigsqcup_{\alpha \in \mathcal{A}_{J}} X_{J, \alpha}=\bigsqcup_{J \subset \Gamma} \bigsqcup_{\beta \in \mathcal{B}_{J}} Y_{J, \beta}
$$

of $\bar{G}$ are said to be compatible if for any $J \subset \Gamma, \alpha \in \mathcal{A}_{J}$, and $\beta \in \mathcal{B}_{J}$ with $X_{J, \alpha} \cap Y_{J, \beta} \neq \emptyset, X_{J, \alpha}$ and $Y_{J, \beta}$ intersect transversally in $Z_{J}$ and $X_{J, \alpha} \cap Y_{J, \beta}$ is irreducible. For two such partitions of $\bar{G}$, and for $K \subset$ $J \subset \Gamma, \alpha \in \mathcal{A}_{J}$, and $\beta \in \mathcal{B}_{J}$, let

$$
\begin{aligned}
\mathcal{A}_{K}^{\alpha} & =\left\{\alpha^{\prime} \in \mathcal{A}_{K}: X_{K, \alpha^{\prime}} \subset \overline{X_{J, \alpha}}, \operatorname{Codim}_{Z_{K}} X_{K, \alpha^{\prime}}=\operatorname{Codim}_{Z_{J}} X_{J, \alpha}\right\}, \\
\mathcal{B}_{K}^{\beta} & =\left\{\beta^{\prime} \in \mathcal{B}_{K}: Y_{K, \beta^{\prime}} \subset \overline{Y_{J, \beta}}, \quad \operatorname{Codim}_{Z_{K}} Y_{K, \beta^{\prime}}=\operatorname{Codim}_{Z_{J}} Y_{J, \beta}\right\} .
\end{aligned}
$$

Proposition 3.3. 1) Any admissible partition of $\bar{G}$ is compatible with the partition of $\bar{G}$ into $G \times G$-orbits;

2) The partitions of $\bar{G}$ into $G_{\delta^{-}}$-stable pieces and into $B^{-} \times B$-orbits are compatible;

3) The partitions of $\bar{G}$ into $B \times B$-orbits and into $B^{-} \times B^{-}$-orbits are compatible.

Proof. Directly from the definition and from Lemma 2.2.

Recall [11, Page 427] that two irreducible subvarieties $X$ and $Y$ of a smooth irreducible variety $Z$ with $X \cap Y \neq \emptyset$ are said to intersect properly in $Z$ if every irreducible component of $X \cap Y$ has dimension equal to $\operatorname{dim} X+\operatorname{dim} Y-\operatorname{dim} Z$. 
Theorem 3.1. Let two compatible partitions of $\bar{G}$ be given as in (23). Then for any $J, K \subset \Gamma$ and $\alpha \in \mathcal{A}_{J}$ and $\beta \in \mathcal{B}_{K}$, if $\overline{X_{J, \alpha}} \cap \overline{Y_{K, \beta}} \neq \emptyset$, then $\overline{X_{J, \alpha}}$ and $\overline{Y_{K, \beta}}$ intersect properly in $\overline{Z_{J \cup K}}$, and

$$
\overline{X_{J, \alpha}} \cap \overline{Y_{K, \beta}}=\bigcup_{\left(\alpha^{\prime}, \beta^{\prime}\right) \in \mathcal{I}_{J \cap K}^{\alpha, \beta}} \overline{X_{J \cap K, \alpha^{\prime}} \cap Y_{J \cap K, \beta^{\prime}}}
$$

is the decomposition of $\overline{X_{J, \alpha}} \cap \overline{Y_{K, \beta}}$ into (distinct) irreducible components, where

$$
\mathcal{I}_{J \cap K}^{\alpha, \beta}=\left\{\left(\alpha^{\prime}, \beta^{\prime}\right) \in \mathcal{A}_{J \cap K}^{\alpha} \times \mathcal{B}_{J \cap K}^{\alpha, \beta}: X_{J \cap K, \alpha^{\prime}} \cap Y_{J \cap K, \beta^{\prime}} \neq \emptyset\right\} .
$$

In particular, $\mathcal{I}_{J \cap K}^{\alpha, \beta} \neq \emptyset$.

Proof. Let $J, K \subset \Gamma, \alpha \in \mathcal{A}_{J}$, and $\beta \in \mathcal{B}_{K}$ be such that $\overline{X_{J, \alpha}} \cap$ $\overline{Y_{K, \beta}} \neq \emptyset$. Regard both $\overline{X_{J, \alpha}}$ and $\overline{Y_{K, \beta}}$ as subvarieties of $\overline{Z_{J \cup K}}$. Since $\overline{Z_{J \cup K}}$ is smooth and irreducible with

$$
\operatorname{dim} Z_{J \cup K}=\operatorname{dim} Z_{J}+\operatorname{dim} Z_{K}-\operatorname{dim} Z_{J \cap K},
$$

every irreducible component of $\overline{X_{J, \alpha}} \cap \overline{Y_{K, \beta}}$ has dimension at least

$$
\begin{aligned}
l & =\operatorname{dim} X_{J, \alpha}+\operatorname{dim} Y_{K, \beta}-\operatorname{dim} Z_{J \cup K} \\
& =\operatorname{dim} Z_{J \cap K}-\operatorname{Codim}_{Z_{J}} X_{J, \alpha}-\operatorname{Codim}_{Z_{K}} Y_{K, \beta} .
\end{aligned}
$$

On the other hand,

$$
\overline{X_{J, \alpha}} \cap \overline{Y_{K, \beta}}=\underbrace{}_{\substack{I \subset J \cap K, \alpha^{\prime} \in \mathcal{A}_{I}, \beta^{\prime} \in \mathcal{B}_{I} \\ X_{I, \alpha^{\prime}} \subset \overline{X_{J, \alpha}}, Y_{I, \beta^{\prime}} \subset \overline{Y_{K, \beta}}}} X_{I, \alpha^{\prime}} \cap Y_{I, \beta^{\prime}} .
$$

For each non-empty intersection on the right hand side of (24),

$$
\begin{aligned}
\operatorname{dim} X_{I, \alpha^{\prime}} \cap Y_{I, \beta^{\prime}} & =\operatorname{dim} Z_{I}-\operatorname{Codim}_{Z_{I}} X_{I, \alpha^{\prime}}-\operatorname{Codim}_{Z_{I}} Y_{I, \beta^{\prime}} \\
& \leqslant \operatorname{dim} Z_{I}-\operatorname{Codim}_{Z_{J}} X_{J, \alpha}-\operatorname{Codim}_{Z_{K}} Y_{K, \beta} \\
& \leqslant \operatorname{dim} Z_{J \cap K}-\operatorname{Codim}_{Z_{J}} X_{J, \alpha}-\operatorname{Codim}_{Z_{K}} Y_{K, \beta} \\
& =l .
\end{aligned}
$$

By Lemma 2.3, every irreducible component of $\overline{X_{J, \alpha}} \cap \overline{Y_{K, \beta}}$ has dimension $l$, and the irreducible components are exactly as described in Theorem 3.1 .

By taking the second admissible partition in Theorem 3.1 to be the one by $G \times G$-orbits, we have the following Corollary 3.1 .

Corollary 3.1. Let a strongly admissible partition of $\bar{G}$ be given as in (21), and let $J \subset \Gamma$ and $\alpha \in \mathcal{A}_{J}$. Then for any $K \subset \Gamma, \overline{X_{J, \alpha}} \cap \overline{Z_{K}} \neq \emptyset$ and $\overline{X_{J, \alpha}}$ and $\overline{Z_{K}}$ intersect properly in $\overline{Z_{J \cup K}}$. Moreover,

$$
\overline{X_{J, \alpha}} \cap \overline{Z_{K}}=\bigcup_{\alpha^{\prime} \in \mathcal{A}_{J \cap K}^{\alpha}} \overline{X_{J \cap K, \alpha^{\prime}}}
$$

is the decomposition of $\overline{X_{J, \alpha}} \cap \overline{Z_{K}}$ into (distinct) irreducible components. 
Corollary 3.2. Let two compatible partitions of $\bar{G}$ be given as in (23). Then for any $J \subset \Gamma$ and $\alpha, \beta \in \mathcal{A}_{J}, X_{J, \alpha} \cap Y_{J, \beta} \neq \emptyset$ if and only if $\overline{X_{J, \alpha}} \cap \overline{Y_{J, \beta}} \neq \emptyset$, and in this case,

$$
\overline{X_{J, \alpha}} \cap \overline{Y_{J, \beta}}=\overline{X_{J, \alpha} \cap Y_{J, \beta}} .
$$

In particular,

$$
\bar{G}=\bigsqcup_{J \subset \Gamma, X_{J, \alpha} \cap Y_{J, \beta} \neq \emptyset} X_{J, \alpha} \cap Y_{J, \beta}
$$

is an admissible partition of $\bar{G}$.

Proof. Take $K=J$ in Theorem 3.1. If $\overline{X_{J, \alpha}} \cap \overline{Y_{J, \beta}} \neq \emptyset$, then $\mathcal{C}_{K, \alpha, \beta}$ consists of one element, namely, $(\alpha, \beta)$. Thus $X_{J, \alpha} \cap Y_{J, \beta} \neq \emptyset$ and (26) holds. The condition on codimensions in Definition 3.1 follows from (25) in the proof of Theorem 3.1.

3.6. We now prove our second main result in this paper. Let

$$
\begin{aligned}
\mathcal{J}=\{(J, x, y, u, v): & J \subset \Gamma,(x, y),(u, v) \in W^{J} \times W, \\
& {\left.[J, x, y] \cap[J, u, v]^{-,-} \neq \emptyset\right\} } \\
=\{(J, x, y, u, v): \quad & J \subset \Gamma, x, u \in W^{J}, y, v \in W, \\
& \left.x \leqslant u, v \leqslant \max \left(y W_{J}\right)\right\}, \\
\mathcal{K}=\{(J, w, x, y): \quad & J \subset \Gamma,(w, x, y) \in W^{J} \times W^{J} \times W, \\
& \left.Z_{J, \delta, w} \cap[J, x, y]^{-,+} \neq \emptyset\right\} \\
=\{(J, w, x, y): \quad & J \subset \Gamma,(w, x, y) \in W^{J} \times W^{J} \times W, \\
& \left.\min \left(W_{J} \delta(w)\right) \leqslant y^{-1} * \delta(x)\right\} .
\end{aligned}
$$

Theorem 3.2. Let $J \subset \Gamma$ and $(x, y),(u, v) \in W^{J} \times W$. Then

$$
[J, x, y] \cap[J, u, v]^{-,-} \neq \emptyset \quad \text { iff } \overline{[J, x, y]} \cap \overline{[J, u, v]^{-,-}} \neq \emptyset,
$$

and in this case,

$$
\overline{[J, x, y] \cap[J, u, v]^{-,-}}=\overline{[J, x, y]} \cap \overline{[J, u, v]^{-,-}} .
$$

In particular,

$$
\bar{G}=\bigsqcup_{(J, x, y, u, v) \in \mathcal{J}}[J, x, y] \cap[J, u, v]^{-,-}
$$

is a strongly admissible partition of $\bar{G}$.

Proof. Assume that $\overline{[J, x, y]} \cap \overline{[J, u, v]^{-,-}} \neq \emptyset$. It follows from Corollary 3.2 that $[J, x, y] \cap[J, u, v]^{-,-} \neq \emptyset$ and (27) holds.

By Corollary 3.2, the partition (28) of $\bar{G}$ is admissible. To show that it is also strongly admissible, let $(J, x, y, u, v) \in \mathcal{J}$ and let $K \subset J$. 
By definition, there exists $z \in W_{J}$ such that $y z=\max \left(y W_{J}\right)$. Set $z^{\prime}=\min \left(z W_{K}\right) \in W^{K}$. Then $x z^{\prime}, u z^{\prime} \in W^{K}$ and

$$
v z^{\prime} \leqslant \max \left(v W_{J}\right) \leqslant \max \left(y W_{J}\right)=y z \leqslant \max \left(y z^{\prime} W_{K}\right) .
$$

Then $\left[K, x z^{\prime}, y z^{\prime}\right] \cap\left[K, u z^{\prime}, v z^{\prime}\right]^{-,-} \neq \emptyset$. By $\left\{3.3\right.$, $\left[K, x z^{\prime}, y z^{\prime}\right] \subset \overline{[J, x, y]}$ and $\left[K, u z^{\prime}, v z^{\prime}\right]^{-,-} \subset \overline{[J, u, v]^{-,-}}$. Therefore

$$
\overline{[J, x, y] \cap[J, u, v]^{-,-}} \cap Z_{K} \supset\left[K, x z^{\prime}, y z^{\prime}\right] \cap\left[K, u z^{\prime}, v z^{\prime}\right]^{-,-} \neq \emptyset .
$$

This shows that the partition (28) is strongly admissible.

Theorem 3.3. Let $J \subset \Gamma, w \in W^{J}$, and $(x, y) \in W^{J} \times W$. Then

$$
Z_{J, \delta, w} \cap[J, x, y]^{-,+} \neq \emptyset \quad \text { iff } \overline{Z_{J, \delta, w}} \cap \overline{[J, x, y]^{-,+}} \neq \emptyset,
$$

and in this case,

$$
\overline{Z_{J, \delta, w}} \cap \overline{[J, x, y]^{-,+}}=\overline{Z_{J, \delta, w} \cap[J, x, y]^{-,+}} .
$$

In particular,

$$
\bar{G}=\bigsqcup_{(J, w, x, y) \in \mathcal{K}} Z_{J, \delta, w} \cap[J, x, y]^{-,+}
$$

is a strongly admissible partition of $\bar{G}$.

Proof. Assume that $\overline{Z_{J, \delta, w}} \cap \overline{[J, x, y]^{-,+}} \neq \emptyset$. It follows from Corollary 3.2 that $Z_{J, \delta, w} \cap[J, x, y]^{-,+} \neq \emptyset$ and (29) holds.

By Corollary [3.2, the partition (30) of $\bar{G}$ is admissible. To show that it is also strongly admissible, let $(J, w, x, y) \in \mathcal{K}$ and let $K \subset J$. By definition, there exists $z \in W_{J}$ such that $y z=\max \left(y W_{J}\right)$. Set $z^{\prime}=\min \left(z W_{K}\right) \in W^{K}$. Then $x z^{\prime} \in W^{K}$. Let $z=z^{\prime} z^{\prime \prime}$ with $z^{\prime \prime} \in W_{K}$. Then $w_{0}^{K} *\left(y z^{\prime}\right)^{-1}=w_{0}^{K} *\left(z^{\prime \prime}(y z)^{-1}\right)=w_{0}^{K} *(y z)^{-1}=w_{0}^{J} * y^{-1}$. Thus

$$
\begin{aligned}
w_{0}^{K} *\left(y z^{\prime}\right)^{-1} * \delta\left(x z^{\prime}\right) & =w_{0}^{J} * y^{-1} * \delta\left(x z^{\prime}\right) \geqslant w_{0}^{J} *\left(y^{-1} * \delta(x)\right) \\
& =\max \left(W_{J}\left(y^{-1} * \delta(x)\right) .\right.
\end{aligned}
$$

Since $\min \left(W_{J} \delta(w)\right) \leqslant y^{-1} * \delta(x)$, one has

$$
\delta(w) \leqslant \max \left(W_{J}\left(y^{-1} * \delta(x)\right) \leqslant w_{0}^{K} *\left(\left(y z^{\prime}\right)^{-1} * \delta\left(x z^{\prime}\right)\right) .\right.
$$

Hence $\min \left(W_{K} \delta(w)\right) \leqslant\left(y z^{\prime}\right)^{-1} * \delta\left(x z^{\prime}\right)$, and

$$
\overline{Z_{J, \delta, w} \cap[J, x, y]^{-,+}} \cap Z_{K} \supset Z_{K, \delta, w} \cap\left[K, x z^{\prime}, y z^{\prime}\right]^{-,+} \neq \emptyset .
$$

This shows that the partition (30) of $\bar{G}$ is strongly admissible.

Remark 3.1. Assume that $\overline{[J, x, y]} \cap \overline{[J, u, v]^{-,-}} \neq \emptyset$, we can also use Proposition 3.1 to prove directly that $[J, x, y] \cap[J, u, v]^{-,-} \neq \emptyset$. Similarly, assume that $\overline{Z_{J, \delta, w}} \cap \overline{[J, x, y]^{-,+}} \neq \emptyset$. One can use Proposition 3.1 to prove directly that $Z_{J, \delta, w} \cap[J, x, y]^{-,+} \neq \emptyset$. We omit the details. 
Consider now the following four strongly admissible partitions of $\bar{G}$ :

$$
\begin{aligned}
\bar{G} & =\bigsqcup_{J \subset \Gamma,(x, y) \in W^{J} \times W}[J, x, y]^{-,+}=\bigsqcup_{J \subset \Gamma, w \in W^{J}} Z_{J, \delta, w} \\
& =\bigsqcup_{(J, x, y, u, v) \in \mathcal{J}}[J, x, y] \cap[J, u, v]^{-,-}=\bigsqcup_{(J, x, y, u, v) \in \mathcal{K}} Z_{J, \delta, w} \cap[J, x, y]^{-,+} .
\end{aligned}
$$

As a direct consequence of Corollary 3.1, we have

Corollary 3.3. Let $J \subset \Gamma$ and let $X$ be any of the subvarieties of $Z_{J}$ appearing in either one of the fours partitions in (31). Then for any $K \subset \Gamma, \bar{X} \cap \overline{Z_{K}} \neq \emptyset$, and $\bar{X}$ and $\overline{Z_{K}}$ intersect properly in $\overline{Z_{J \cup K}}$.

Corollary 3.1 also allows us to describe the irreducible components of the non-empty intersections in Corollary 3.3 .

Corollary 3.4. 1) For any $J \subset \Gamma,(x, y) \in W^{J} \times W$, and $K \subset \Gamma$,

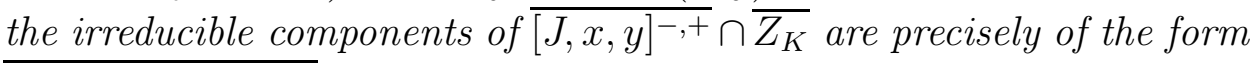
$\overline{[J \cap K, x u, y u]^{-,+}}$, where $u \in W_{J} \cap W^{J \cap K}$ and $l(y u)=l(y)+l(u)$.

2) For any $J \subset \Gamma, w \in W^{J}$, and $K \subset \Gamma$, the irreducible components of $\overline{Z_{J, \delta, w}} \cap \overline{Z_{K}}$ are precisely of the form $\overline{Z_{J \cap K, \delta, w^{\prime}}}$ with $w^{\prime} \in W^{J \cap K} \cap$ $\operatorname{Min}\left(C_{J}(w)\right)$.

3) For any $(J, x, y, u, v) \in \mathcal{J}$ and $K \subset \Gamma$, the irreducible components

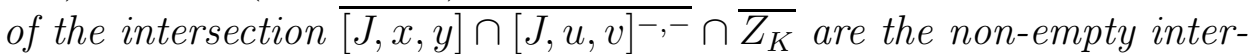
sections of irreducible components of $\overline{[J, x, y]} \cap \overline{Z_{K}}$ and the irreducible components of $\overline{[J, u, v]^{-,-}} \cap \overline{Z_{K}}$.

4) For any $(J, w, x, y) \in \mathcal{K}$ and $K \subset \Gamma$, the irreducible components of $\overline{Z_{J, \delta, w} \cap[J, x, y]^{-,+}} \cap \overline{Z_{K}}$ are the non-empty intersections of irreducible components of $\overline{Z_{J, \delta, w}} \cap \overline{Z_{K}}$ and the irreducible components of $\overline{[J, x, y]^{-,+}} \cap \overline{Z_{K}}$.

Remark 3.2. Corollary 3.3 and Corollary 3.4 in the case of the intersections $\overline{[J, x, y]^{-,+}} \cap \overline{Z_{K}}$ have also been obtained by M. Brion in [1] (using $B \times B^{-}$-orbits instead of $B^{-} \times B$-orbits).

Applying Theorem 3.1, we have

Corollary 3.5. 1) If $J, K \subset \Gamma$ and $(x, y) \in W^{J} \times W,(u, v) \in W^{K} \times W$ are such that $\overline{[J, x, y]} \cap \overline{[K, u, v]^{-,-}} \neq \emptyset$, then $\overline{[J, x, y]}$ and $\overline{[K, u, v]^{-,-}}$ intersect properly in $\overline{Z_{J \cup K}}$, and the irreducible components of $\overline{[J, x, y]} \cap$ $\overline{[K, u, v]^{-,-}}$are the non-empty intersections of irreducible components of $\overline{[J, x, y]} \cap \overline{Z_{J \cap K}}$ and the irreducible components of $\overline{[K, u, v]^{-,-}} \cap \overline{Z_{J \cap K}}$. 2) If $J, K \subset \Gamma$ and $(w, x, y) \in W^{J} \times W^{K} \times W$ are such that $\overline{Z_{J, \delta, w}} \cap$ $\overline{[K, x, y]^{-,+}} \neq \emptyset$, then $\overline{Z_{\mathcal{C}, \delta, w}}$ and $\overline{[K, x, y]^{-,+}}$intersect properly in $\overline{Z_{J \cup K}}$, and the irreducible components of $\overline{Z_{J, \delta, w}} \cap \overline{[K, x, y]^{-,+}}$are the non-empty intersections of irreducible components of $\overline{Z_{J, \delta, w}} \cap \overline{Z_{J \cap K}}$ and the irreducible components of $\overline{[K, x, y]^{-,+}} \cap \overline{Z_{J \cap K}}$. 


\section{A generalization of Deligne-Lusztig varieties}

4.1. Let the ground field be an algebraically closed field in positive characteristic. Let $F: G \rightarrow G$ be a Frobenius map. We may choose a Borel subgroup $B$ and a maximal torus $H$ in such a way that $F(B)=B$ and $F(H)=H$. Then $F$ induces an automorphism on $W$ which we still denote by $F$. Set $G_{F}=\{(g, F(g)): g \in G\} \subset G \times G$. Let $\mathcal{C}=\left(J, J^{\prime}, c, L\right)$ be an admissible quadruple as in 2.1. For $w \in W^{J}$, define $Z_{\mathcal{C}, F, w}=G_{F}(B w, B) . R_{\mathcal{C}}$. By [14],

1) $Z_{\mathcal{C}, F, w}$ is a single $G_{F-\text { orbit. }}$

2) $Z_{\mathcal{C}}=\bigsqcup_{w \in W^{J}} Z_{\mathcal{C}, F, w}$.

3) $\overline{Z_{\mathcal{C}, F, w}}=\bigsqcup Z_{\mathcal{C}, F, w^{\prime}}$, where $w^{\prime}$ runs over elements in $W^{J}$ such that $\delta^{-1}(F(u)) w^{\prime} u^{-1} \leqslant w$ for some $u \in W_{J}$.

4.2. We now consider the intersection of a $G_{\delta^{-}}$stable piece and a $G_{F^{-}}$ orbit in $Z_{\mathcal{C}}$. In the special case where $Z_{\mathcal{C}}=G / B \times G / B$ and $\delta$ is identity map, $Z_{\mathcal{C}, \delta, 1} \cap Z_{\mathcal{C}, F, w^{\prime}}$ are just the Deligne-Lusztig varieties [5]. It is also worth mentioning that in general Deligne-Lusztig varieties are not irreducible and not Frobenius split. See [9].

Proposition 4.1. For any $w, w^{\prime} \in W^{J}$, one has $Z_{\mathcal{C}, \delta, w} \cap Z_{\mathcal{C}, F, w^{\prime}} \neq \emptyset$, and $\overline{Z_{\mathcal{C}, \delta, w}} \cap \overline{Z_{\mathcal{C}, F, w^{\prime}}}=\overline{Z_{\mathcal{C}, \delta, w} \cap Z_{\mathcal{C}, F, w^{\prime}}}$.

Proof. Notice that $R_{\mathcal{C}} \in Z_{\mathcal{C}, \delta, 1} \cap Z_{\mathcal{C}, F, 1}$. Hence $\overline{Z_{\mathcal{C}, \delta, w}} \cap \overline{Z_{\mathcal{C}, F, w^{\prime}}} \neq \emptyset$. Notice that $\operatorname{Lie}\left(G_{F}\right)=(\operatorname{Lie}(G), 0)$. Thus $\operatorname{Lie}\left(G_{\delta}\right)+\operatorname{Lie}\left(G_{F}\right)=\operatorname{Lie}(G) \oplus$ $\operatorname{Lie}(G)$. So $\overline{Z_{\mathcal{C}, \delta, w}}$ intersects transversally with $\overline{Z_{\mathcal{C}, F, w^{\prime}}}$. Therefore each irreducible component of $\overline{Z_{\mathcal{C}, \delta, w}} \cap \overline{Z_{\mathcal{C}, F, w^{\prime}}}$ is of dimension $\operatorname{dim} \overline{Z_{\mathcal{C}, \delta, w}}+$ $\operatorname{dim} \overline{Z_{\mathcal{C}, F, w^{\prime}}}-\operatorname{dim} Z_{\mathcal{C}}$. On the other hand, $\overline{Z_{\mathcal{C}, \delta, w}} \cap \overline{Z_{\mathcal{C}, F, w^{\prime}}}$ is the union of subvarieties $Z_{\mathcal{C}, \delta, x} \cap Z_{\mathcal{C}, F, y}$, where $x$ and $y$ run over elements in $W^{J}$ such that $Z_{\mathcal{C}, \delta, x} \subset \overline{Z_{\mathcal{C}, \delta, w}}$ and $Z_{\mathcal{C}, F, y} \subset \overline{Z_{\mathcal{C}, F, w^{\prime}}}$. For such $x$ and $y$,

$$
\begin{aligned}
\operatorname{dim}\left(Z_{\mathcal{C}, \delta, x} \cap Z_{\mathcal{C}, F, y}\right) & =\operatorname{dim} Z_{\mathcal{C}, \delta, x}+\operatorname{dim} Z_{\mathcal{C}, F, y}-\operatorname{dim} Z_{\mathcal{C}} \\
& \leqslant \operatorname{dim} \overline{Z_{\mathcal{C}, \delta, w}}+\operatorname{dim} \overline{Z_{\mathcal{C}, F, w^{\prime}}}-\operatorname{dim} Z_{\mathcal{C}}
\end{aligned}
$$

with equality holds only when $\operatorname{dim} Z_{\mathcal{C}, \delta, x}=\operatorname{dim} \overline{Z_{\mathcal{C}, \delta, w}}$ and $\operatorname{dim} Z_{\mathcal{C}, F, y}=$ $\operatorname{dim} \overline{Z_{\mathcal{C}, F, w^{\prime}}}$, i.e., $x=w$ and $y=w^{\prime}$. Therefore the irreducible components of $\overline{Z_{\mathcal{C}, \delta, w}} \cap \overline{Z_{\mathcal{C}, F, w^{\prime}}}$ are precisely the closure $\bar{Y}$, where $Y$ is an irreducible component of $Z_{\mathcal{C}, \delta, w} \cap Z_{\mathcal{C}, F, w^{\prime}}$. In particular, $Z_{\mathcal{C}, \delta, w} \cap Z_{\mathcal{C}, F, w^{\prime}} \neq \emptyset$ and $\overline{Z_{\mathcal{C}, \delta, w}} \cap \overline{Z_{\mathcal{C}, F, w^{\prime}}}=\overline{Z_{\mathcal{C}, \delta, w} \cap Z_{\mathcal{C}, F, w^{\prime}}}$.

Remark 4.1. The Proposition 4.1 can also be generalized to $\bar{G}$. We omit the details.

\section{Appendix}

Recall that $W$ is the Weyl group of $G$. We now prove some properties of the operations $*, \triangleleft, \triangleright$ on $W$ as defined in Section 2.5. In fact, many properties also holds for arbitrary Coxeter groups. See [15]. 
Lemma 5.1. [12, Lemma 3.3] For any $x, y \in W$,

1) $x * y \in W$ is the unique maximal element in the set $\{u y: u \leqslant x\}$ as well as in the set $\{x v ; v \leqslant y\}$. Moreover, $x * y=x_{1} y=x y_{1}$, where $x_{1} \leqslant x, y_{1} \leqslant y$ and $l(x * y)=l\left(x_{1}\right)+l(y)=l(x)+l\left(y_{1}\right)$;

2) $x \triangleright y \in W$ is the unique minimal element in the set $\{u y: u \leqslant x\}$, and $x \triangleright y=x_{1} y$ with $x_{1} \leqslant x$ and $l(x \triangleright y)=l(y)-l\left(x_{1}\right)$;

3) $x \triangleleft y \in W$ is the unique minimal element in the set $\{x v ; v \leqslant y\}$, and $x \triangleleft y=x y_{1}$ with $y_{1} \leqslant y$ and $l(x \triangleleft y)=l(x)-l\left(y_{1}\right)$.

Lemma 5.2. Let $x, x^{\prime}, y, y^{\prime} \in W$. If $x \leqslant x^{\prime}$ and $y \leqslant y^{\prime}$, then

$$
x * y \leqslant x^{\prime} * y^{\prime}, \quad x^{\prime} \triangleright y \leqslant x \triangleright y^{\prime}, \quad \text { and } \quad x \triangleleft y^{\prime} \leqslant x^{\prime} \triangleleft y .
$$

Proof. It follows from

$$
B(x * y) B \subset B x B y B \subset \overline{B x^{\prime} B} \overline{B y^{\prime} B} \subset \overline{B x^{\prime} B y^{\prime} B}=\overline{B\left(x^{\prime} * y^{\prime}\right) B},
$$

that $x * y \leqslant x^{\prime} * y^{\prime}$. Similarly, since

$B\left(x \triangleright y^{\prime}\right) B^{-} \subset B x B y^{\prime} B^{-} \subset \overline{B x^{\prime} B} \overline{B y B^{-}} \subset \overline{B x^{\prime} B y B^{-}}=\overline{B\left(x^{\prime} \triangleright y\right) B^{-}}$, one has $x^{\prime} \triangleright y \leqslant x \triangleright y^{\prime}$. Similarly, $x \triangleleft y^{\prime} \leqslant x^{\prime} \triangleleft y$.

Lemma 5.3. For any $x, y, z \in W$,

1) $x \triangleright y=\left(x *\left(y w_{0}\right)\right) w_{0}$ and $x \triangleleft y=w_{0}\left(\left(w_{0} x\right) * y\right)$;

2) $(x \triangleleft y)^{-1}=y^{-1} \triangleright x^{-1}$ and $(x * y)^{-1}=y^{-1} * x^{-1}$;

3) $x \triangleright y \leqslant z$ if and only if $y \leqslant x^{-1} * z$;

4) $y \triangleleft x \leqslant z$ if and only if $y \leqslant z * x^{-1}$;

5) $(x \triangleright y) \triangleleft z=x \triangleright(y \triangleleft z)$.

Proof. 1) Since

$$
\begin{aligned}
\overline{B(x \triangleright y) B^{-}} & =\overline{B x B y B^{-}}=\overline{B x B y w_{0} B w_{0}}=\overline{B\left(x *\left(y w_{0}\right)\right) B w_{0}} \\
& =\overline{B\left(x *\left(y w_{0}\right)\right) w_{0} B^{-}},
\end{aligned}
$$

one has $x \triangleright y=x \triangleright\left(y w_{0}\right) w_{0}$. Similarly, $x \triangleleft y=w_{0}\left(\left(w_{0} x\right) * y\right)$.

2) Let $\tau$ be the inverse map of $G$. Then $(x \triangleleft y)^{-1}=y^{-1} \triangleright x^{-1}$ follows by applying $\tau$ to $\overline{B^{-}(x \triangleleft y) B}=\overline{B^{-} x B y B}$. Similarly, $(x * y)^{-1}=y^{-1} * x^{-1}$.

3) Since $y \in\left\{u(x \triangleright y): u \leqslant x^{-1}\right\}, y \leqslant x^{-1} *(x \triangleright y)$. If $x \triangleright y \leqslant z$, then $y \leqslant x^{-1} * z$ by Lemma 5.2. Similarly, $z \in\left\{u\left(x^{-1} * z\right): u \leqslant x\right\}$, so $x \triangleright\left(x^{-1} * z\right) \leqslant z$. If $y \leqslant x^{-1} * z$, then by Lemma [5.2, $x \triangleright y \leqslant$ $x \triangleright\left(x^{-1} * z\right) \leqslant z$.

Part 4) can be proved in the same way as part 3).

5) Since

$$
\begin{aligned}
B((x \triangleright y) \triangleleft z) B^{-} & \subset B(x \triangleright y) B^{-} z B^{-} \subset B x B y B^{-} z B^{-} \\
& \subset \overline{B x B} \overline{B y B^{-} z B^{-}}=\overline{B x B} \overline{B(y \triangleleft z) B^{-}} \\
& \subset \overline{B x B(y \triangleleft z) B^{-}}=\overline{B(x \triangleright(y \triangleleft z)) B^{-},}
\end{aligned}
$$

one sees that $(x \triangleright y) \triangleleft z \geqslant x \triangleright(y \triangleleft z)$. Similarly, one shows that $(x \triangleright y) \triangleleft z \leqslant x \triangleright(y \triangleleft z)$. Thus $(x \triangleright y) \triangleleft z=x \triangleright(y \triangleleft z)$. 
Lemma 5.4. For $J, J^{\prime} \subset \Gamma, x \in W, y \in W^{J}$ and $z \in{ }^{J} W$, one has $x \triangleright y \in W^{J}, z \triangleleft x \in{ }^{J} W$, and

$$
w_{0}^{J^{\prime}} \triangleright x \triangleleft w_{0}^{J}=\min \left(W_{J^{\prime}} x W_{J}\right), \quad w_{0}^{J^{\prime}} * x * w_{0}^{J}=\max \left(W_{J^{\prime}} x W_{J}\right) .
$$

Proof. By Lemma 5.1, $x \triangleright y=x_{1} y$ with $x_{1} \leqslant x$ and $l(x \triangleright y)=$ $l(y)-l\left(x_{1}\right)$. By [12, 3.5], $x_{1} y \in W^{J}$. Similarly one has $z \triangleleft x \in{ }^{J} W$. It is clear from the definitions that

$$
w_{0}^{J^{\prime}} \triangleright x=\min \left(W_{J^{\prime}} x\right) \in{ }^{J^{\prime}} W \quad \text { and } \quad x \triangleleft w_{0}^{J}=\min \left(x W_{J}\right) \in W^{J} .
$$

Thus $w_{0}^{J^{\prime}} \triangleright x \triangleleft w_{0}^{J} \in{ }^{J^{\prime}} W^{J}$. By Lemma 5.1, $w_{0}^{J^{\prime}} \triangleright x \triangleleft w_{0}^{J} \in W_{J^{\prime}} x W_{J}$. Thus $w_{0}^{J^{\prime}} \triangleright x \triangleleft w_{0}^{J}=\min \left(W_{J^{\prime}} x W_{J}\right)$. Similarly, $w_{0}^{J^{\prime}} * x * w_{0}^{J}=\max \left(W_{J^{\prime}} x W_{J}\right)$.

Combining Lemma 5.4 with Lemma 5.3 4), we have the following consequence.

Lemma 5.5. For any $J \subset \Gamma$ and $x, y \in W, x \leqslant \max \left(y W_{J}\right)$ if and only if $\min \left(x W_{J}\right) \leqslant y$.

The following Lemma 5.6 can be found in [4, 18].

Lemma 5.6. For $x, y \in W$, the following conditions are equivalent:

1) $B x B \subset B y B$;

2) $B^{-} y B \subset B^{-} x B$;

3) $\left(B^{-} x B\right) \cap(B y B) \neq \emptyset$;

4) $\overline{B^{-} x B} \cap \overline{B y B} \neq \emptyset$;

5) $x \leqslant y$.

The following result will be used several times in our paper.

Lemma 5.7. For $x, y, u, v \in W$, the following conditions are equivalent:

1) $(B x B y B) \cap\left(B^{-} u B v B\right) \neq \emptyset$;

2) $\overline{B x B y B} \cap\left(B^{-} u B v B\right) \neq \emptyset$;

3) $(B x B y B) \cap \overline{B^{-} u B v B} \neq \emptyset$;

4) $\overline{B x B y B} \cap \overline{B^{-} u B v B} \neq \emptyset$;

5) $u \triangleleft v \leqslant x * y$.

6) $u \leqslant x * y * v^{-1}$.

Proof. Clearly 1) implies 2) and 3), 2) or 3) implies 4), 4) implies 5 ) by Lemma 5.6 and 5) is equivalent to 6 ) by Lemma 5.3. It suffices to show that 5) implies 1).

Suppose that $u \triangleleft v \leqslant x * y$. Then $(B(x * y) B) \cap\left(B^{-}(u \triangleleft v) B\right) \neq \emptyset$ by Lemma 5.6. Since $B(x * y) B \subset B x B y B$ and $B^{-}(u \triangleleft v) B \subset B^{-} u B v B$, we have $(B x B y B) \cap\left(B^{-} u B v B\right) \neq \emptyset$. Hence 5$)$ implies 1). 


\section{ACKNOWLEDGMENTS}

We thank J. Starr and J. F. Thomsen for some helpful discussions. The first author is partially supported by HKRGC grants 601409 and DAG08/09.SC03. The second author is partially supported by HKRGC grants 703405 and 703707 .

\section{REFERENCES}

[1] M. Brion, The behavior at infinity of the Bruhat decomposition, Comment. Math. Helv. 73 (1998), 137 - 174.

[2] C. De Concini and C. Procesi, Complete symmetric varieties, in Invariant theory (Montecatini, 1982), Lecture Notes in Math. 996 (1983), 1-44.

[3] C. De Concini and T. Springer, Compactification of symmetric spaces, Transformation Groups 4 (1999), 273-300.

[4] V. Deodhar, On some geometric aspects of Bruhat orderings, I. A finer decomposition of Bruhat cells, Invent. Math. 79 (1985), 499 - 511.

[5] P. Deligne and G. Lusztig, Representations of reductive groups over finite fields, Ann. of Math. (2) 103 (1976), no. 1, 103-161.

[6] S. Evens and J.-H. Lu, On the variety of Lagrangian subalgebras, II, Ann. Scient. Ecol. Norm. Sup., 39 (2) (2006), 347 - 379.

[7] S. Evens and J.-H. Lu, Poisson geometry of the Grothendieck resolution of a complex semisimple group, Moscow Mathematical Journal 7 (4) (special volume in honor of V. Ginzburg's 50'th birthday) (2007), 613 - 642.

[8] S. Fomin and A. Zelevinsky, Double Bruhat cells and total positivity, J. Amer. Math. Soc. 12 (1999), no. 2, 335-380.

[9] S. H. Hansen, Canonical bundles of Deligne-Lusztig varieties, Manuscripta Math. 98 (1999), 363-375.

[10] J. Harris, Algebraic geometry, Graduate Texts in Mathematics, 133 SpringerVerlag, 1992.

[11] R. Hartshorne, Algebraic geometry, Graduate Texts in Mathematics, 52, Springer-Verlag, 1977.

[12] X. He, The G-stable pieces of the wonderful compactification, Tran. of AMS 359 (7) (2007), 3005 - 3024.

[13] X. He, Minimal length elements in some double cosets of Coxeter groups, Adv. Math. 215 (2007), 469 - 503.

[14] X. He, G-stable pieces and partial flag varieties, Representation Theory, Contemp. Math., vol. 478, Amer. Math. Soc., Providence, RI, 2009, pp. 6170.

[15] X. He, A subalgebra of 0-Hecke algebra, to appear in J. Algebra, 2009.

[16] X. He and J. F. Thomsen, Geometry of $B \times B$-orbit closures in equivariant embeddings, Adv. Math. 216 (2) (2007), 626-646.

[17] X. He and J. F. Thomsen, Frobenius splitting and geometry of G-Schubert varieties, Adv. Math. 219 (5) (2008), 1469 - 1512.

[18] D. Kazhdan and G. Lusztig, Schubert varieties and Poincaré duality, Proc. Symp. Pure Math. 36, Amer. Math. Soc. (1980), pp. 185-203.

[19] M. Kogan and A. Zelevinsky, On symplectic leaves and integrable systems in standard complex semisimple Poisson-Lie groups, Int. Math. Res. Not (32) (2002), 1685-1702.

[20] J. Lu and M. Yakimov, Partitions of the wonderful group compactification, Trans. Groups, 12 (4) (2007), 695 - 723.

[21] J. Lu and M. Yakimov, Group orbits and regular partitions of Poisson manifolds, Comm. Math. Phys., 283(3), 729 - 748 (2008).

[22] G. Lusztig, Parabolic character sheaves I, Moscow Math J. 4 (2004), 153-179. 
[23] G. Lusztig, Parabolic character sheaves II, Moscow Math J. 4 (2004), 869-896.

[24] R. W. Richardson, Intersections of double cosets in algebraic groups, Indagationes Mathematicae, Volume 3, Issue 1, (1992), 69-77.

[25] R. W. Richardson and T. A. Springer, The Bruhat order on symmetric spaces, Geom. Dedic. 35 (1990), 389 - 436.

[26] R. Rouquier, Weyl groups, affine Weyl groups and reflection groups, Representations of reductive groups, 21-39, Publ. Newton Inst., Cambridge Univ. Press, Cambridge, 1998.

[27] T. A. Springer, Intersection cohomology of $B \times B$-orbit closures in group compactifications, with an appendix by W. van der Kallen. Special issue in celebration of Claudio Procesi's 60th birthday, J. Algebra 258 (2002), 71-111.

[28] T. A. Springer, An extension of Bruhat's Lemma, J. Alg. 313 (2007), 417-427.

Department of Mathematics, Hong Kong University of Science And Technology, Clear Water Bay, Hong Kong

E-mail address: maxhhe@ust.hk

Department of Mathematics, The University of Hong Kong, PokfuLAM ROAD, HONG KONG

E-mail address: jhlu@maths.hku.hk 